\title{
Computational analysis of calcium signaling and membrane electrophysiology in cerebellar Purkinje neurons associated with ataxia
}

Sherry-Ann Brown and Leslie M Loew ${ }^{*}$

\begin{abstract}
Background: Mutations in the smooth endoplasmic reticulum (sER) calcium channel Inositol Trisphosphate Receptor type 1 (IP3R1) in humans with the motor function coordination disorders Spinocerebellar Ataxia Types 15 and 16 (SCA15/16) and in a corresponding mouse model, the IP3R1 delta18/delta18 mice, lead to reduced IP3R1 levels. We posit that increasing IP3R1 sensitivity to IP3 in ataxias with reduced IP3R1 could restore normal calcium response. On the other hand, in mouse models of the human polyglutamine (polyQ) ataxias, SCA2, and SCA3, the primary finding appears to be hyperactive IP3R1-mediated calcium release. It has been suggested that the polyQ SCA1 mice may also show hyperactive IP3R1. Yet, SCA1 mice show downregulated gene expression of IP3R1, Homer, metabotropic glutamate receptor (mGluR), smooth endoplasmic reticulum Ca-ATP-ase (SERCA), calbindin, parvalbumin, and other calcium signaling proteins.

Results: We create a computational model of pathological alterations in calcium signaling in cerebellar Purkinje neurons to investigate several forms of spinocerebellar ataxia associated with changes in the abundance, sensitivity, or activity of the calcium channel IP3R1. We find that increasing IP3R1 sensitivity to IP3 in computational models of SCA15/16 can restore normal calcium response if IP3R1 abundance is not too low. The studied range in IP3R1 levels reflects variability found in human and mouse ataxic models. Further, the required fold increases in sensitivity are within experimental ranges from experiments that use IP3R1 phosphorylation status to adjust its sensitivity to IP3. Results from our simulations of polyglutamine SCAs suggest that downregulation of some calcium signaling proteins may be partially compensatory. However, the downregulation of calcium buffer proteins observed in the SCA1 mice may contribute to pathology. Finally, our model suggests that the calcium-activated voltage-gated potassium channels may provide an important link between calcium metabolism and membrane potential in Purkinje cell function.

Conclusion: Thus, we have established an initial platform for computational evaluation and prediction of ataxia pathophysiology. Specifically, the model has been used to investigate SCA15/16, SCA1, SCA2, and SCA3. Results suggest that experimental studies treating mouse models of any of these ataxias with appropriately chosen peptides resembling the C-terminal of IP3R1 could adjust receptor sensitivity, and thereby modulate calcium release and normalize IP3 response. In addition, the model supports the hypothesis of IP3R1 supersensitivity in SCA1.
\end{abstract}

Keywords: Model, IP3R, Homer, CAG repeat instability, KCa channels, Ataxia, Computational, BK channel, Spinocerebellar, Virtual cell

\footnotetext{
*Correspondence: les@volt.uchc.edu

Richard D. Berlin Center for Cell Analysis \& Modeling, University of

Connecticut Health Center, 400 Farmington Ave, Farmington CT 06030, USA
} 


\section{Background}

Degeneration of the cerebellum or dysfunction of the Purkinje neurons leads to lack of motor coordination, or ataxia, and impaired motor learning [1]. There are several forms of hereditary ataxia, but the genetic basis of many ataxias is unknown. The IP3R1 calcium channel has been implicated in ataxic mice [2-11] and very recently in humans with ataxia [3,9,12-17] (see reviews [18,19]). A portion of IP3R1 is represented in Figure 1a and, along with Table 1, will be referenced in various sections of this computational study, which focuses on a handful of ataxias involving this key calcium handling protein. Spinocerebellar ataxia 15 (SCA15) and Spinocerebellar ataxia 16 (SCA16) are two forms of autosomal dominant pure cerebellar ataxia that involve enormous heterozygous deletions or missense mutations in IP3R1 [15-17] (see Figure 1b and Table 1). However, pathophysiology of SCA15/16 has not been studied extensively. The ITPR $1^{\Delta 18 / \mathrm{wt}}$ and ITPR $1^{\Delta 18 / \Delta 18}$ mice (mouse models for SCA15/16 [3]) possess heterozygous and homozygous, respectively, in-frame 18 bp deletions in exon 36 of the gene that encodes IP3R1. These mice showed reduced levels of cerebellar IP3R1 on Western blot and with immunofluorescence [3] (Figure 1b). IP3R $^{+/-}$mice also exhibit motor discoordination on the rotor rod test, and IP3R $1^{-/-}$mice show lack of balance when upright $[2,5]$. Various other forms of ataxia in mice and humans with causal mutations in genes other than IP3R1 also show reduced IP3R1 protein levels $[11,14,20]$. Taken together, these findings suggest that haploinsufficiency of IP3R1 may contribute to cerebellar ataxia [12].

The mechanism of pathology is different in polyglutamine ataxias. Humans with spinocerebellar Ataxia 1
(SCA1), spinocerebellar ataxia 2 (SCA2), and spinocerebellar ataxia 3 (SCA3), and their corresponding mouse models, have large numbers of unstable polyglutamine (polyQ, CAG) repeats in the Ataxin-1 [23,24], Ataxin-2 [25], and Ataxin-3 [26-28] proteins, respectively. All three mouse models show increased calcium release in response to IP3, relative to wild type mice $[6,8,10]$, with results from SCA2 and SCA3 suggesting a mechanism involving supersensitive IP3R1 [6,10,18]. SCA1 and SCA3 mice have reduced expression of IP3R1 and other glutamatergic signaling proteins [29], in addition to increased IP3R1-mediated calcium release, relative to wild type mice [8]. This paradox is yet to be explained [30]. Gardner suggests that SCA15/16 in humans found to have actual mutations in ITPR1, the gene that encodes the IP3R1 protein, be termed ITPR1-associated ataxia [31]. However, we have termed a subset of ataxias, not just with reduced IP3R1 but also with supersensitive IP3R1, and regardless of the mutated gene (e.g., ITPR1, Ataxin-1, Ataxin-2, Ataxin-3) as 'IP3R1-associated ataxias' [32].

A prior modeling study by Hernjak et al. explores the significance of experimental observations in normal cerebellar Purkinje neurons, which show markedly lower sensitivity and higher abundance of IP3R1 relative to other cell types [33-35]. This is the opposite of what is seen in SCA3 and suggested in SCA1. Hernjak et al. demonstrated that the normally high levels of IP3R1 ensure robust calcium signals in an individual spine, and that the normally low sensitivity of IP3R1 to IP3 confines the calcium transients to the stimulated spine, without spillover to adjacent spines [35]. Thus, they argued that
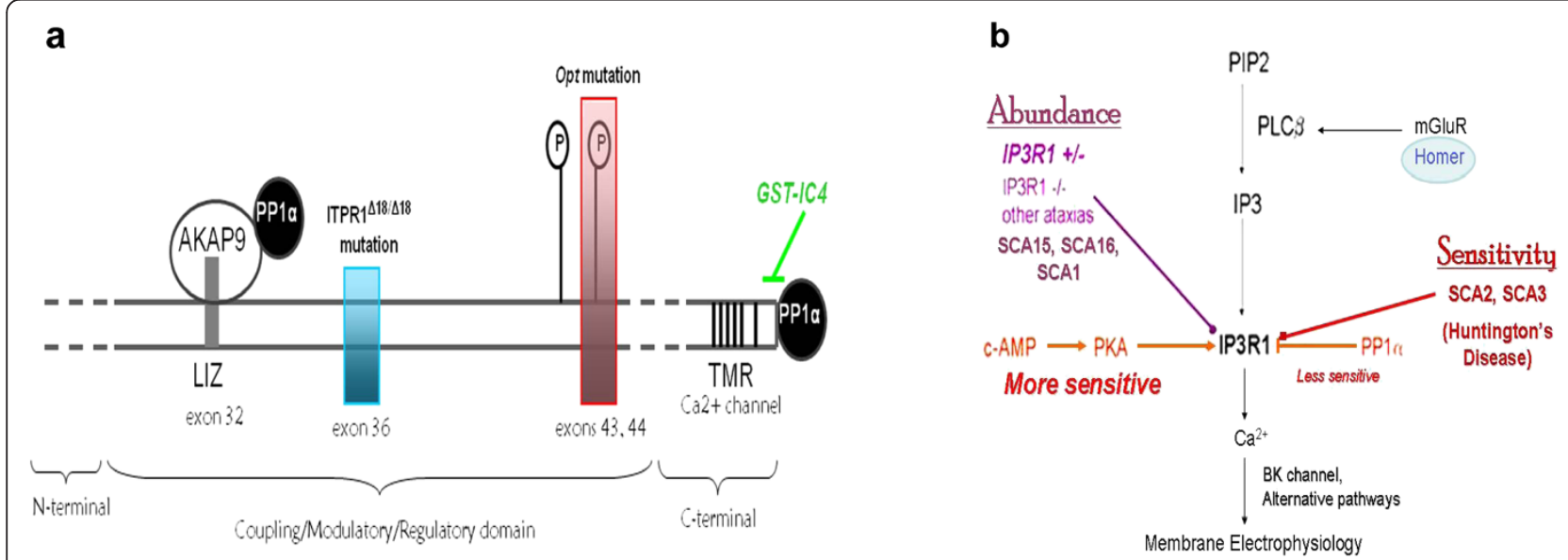

Figure 1 Schematics relevant to this study. a, A portion of the regulatory domain of IP3R1 and the C-terminal, adapted from [21,22], with the PP1 binding motif and mutations for ITPR118/18 and ITPR1op1/opt mice. $\mathbf{b}$, Summary of various aspects of this study. IP3R1 is the center of this study, involved in phosphoinositol signaling, calcium release, membrane potential modulation; the dysregulation of any of these functions may lead to ataxia. 
Table 1 Ataxic mouse models relevant to this study

\begin{tabular}{|c|c|c|c|c|}
\hline Ataxia model & $\begin{array}{l}\text { Mutated gene } \\
\text { product }\end{array}$ & Protein name & Pathology & $\begin{array}{l}\text { Compensation *Observed/ } \\
* * \text { Suggested Therapeutic }\end{array}$ \\
\hline BK -/- & BK & $\begin{array}{l}\text { Large conductance calcium-activated } \\
\text { voltage-gated potassium channel }\end{array}$ & Altered Precision Timing & - \\
\hline IP3R1 -/- & IP3R1 & Inositol triphosphate receptor 1 & No IP3R1 & - \\
\hline IP3R1 +/- & IP3R1 & Inositol triphosphate receptor 1 & $\mathrm{R}$ & $S^{* *}$ \\
\hline IP3R1 delta18/delta18 & IP3R1 & Inositol triphosphate receptor 1 & $\mathrm{R}$ & $S^{* *}$ \\
\hline IP3R1 opt/opt & IP3R1 & Inositol triphosphate receptor 1 & $\mathrm{R}$ & Equivocal \\
\hline SCA15 & IP3R1 & Inositol triphosphate receptor 1 & $\mathrm{R}$ & $S^{* *}$ \\
\hline SCA16 & IP3R1 & Inositol triphosphate receptor 1 & $\mathrm{R}$ & $S^{* *}$ \\
\hline PMCA -/- & PMCA & Plasma Membrane Calcium ATPase & No PCMA & $\mathrm{R}^{*}$ \\
\hline SCA1 & Ataxin1 & Ataxin-1 & $P, A, I$ & $\mathrm{R}^{*}$ \\
\hline SCA2 & Ataxin2 & Ataxin-2 & $P, A, S, I$ & $R^{* *}$ \\
\hline SCA3 & Ataxin3 & Ataxin-3 & $\mathrm{P}, \mathrm{A}, \mathrm{S}, \mathrm{I}$ & $\mathrm{R}^{*}$ \\
\hline
\end{tabular}

$\mathrm{P}=$ Polyglutamine expansion; $\mathrm{S}=$ Supersensitive IP3R1; A = Association of mutant Ataxin with IP3R1 C-terminal; R= Reduced IP3R1 abundance; I= Increased calcium release.

IP3R1 abundance and sensitivity have independent effects on calcium signals and that changes in one do not appear to appropriately compensate for abnormalities in the other. In this work, however, we explore this argument by computationally adjusting IP3R1 sensitivity to IP3 in SCA15/16 (in the context of reduced IP3R1 levels) to see if interventions of this type can actually restore normal calcium response indistinguishable from wild type. We also suggest that, in polyglutamine SCAs with hyperactive IP3R1, experimentally observed downregulation of IP3R1 and other calcium signaling proteins (but not calcium buffers) could serve to partially restore normal calcium transients in ataxias.

We test our hypotheses in a novel version of a recently developed computational model that links biochemistry with electrophysiology [36]. We simulate the application of different experimentally available peptides that resemble various portions of the C-terminal of IP3R1, in order to model competitive inhibition of the binding of mutant ataxin proteins. Improvements are made to the model, resulting in an ability to look at changes in membrane potential in response to IP3R1-mediated calcium release or supralinear calcium transients. We show that adjusting IP3R1 sensitivity to IP3, using peptides resembling the C-terminal of IP3R1, could potentially stabilize calcium, without altering fine-tuning of supralinear calcium release required for normal Purkinje neuron function and long term-depression. The model also demonstrates that decreased levels of IP3R1 and various other calcium signaling proteins (except for calcium buffers) experimentally observed in SCA1 mice may partially compensate for the IP3R1 supersensitivity suggested by Liu et al. [10]. Finally, we link IP3R1 activity with the influence of calcium on membrane electrophysiology. Results from our study may provide further insight into the roles that abundance and sensitivity of the calcium channel IP3R1 play in cerebellar function and coincident detection at IP3R1. Results may also provide a foundation for future studies on SCA1, SCA2, SCA3, SCA15, SCA16, and other IP3R1-associated ataxias, which all involve aberrant calcium signaling.

\section{Methods}

Merged biochemical and electrophysiological modeling

Our analysis of IP3 and calcium dynamics in SCA Purkinje neurons is based on several computational studies of normal physiology from our lab [32,35,36]; these will be briefly described.

The cerebellar Purkinje neuron geometry is complex. In order to combine detailed biochemistry with electrophysiology in tractable Virtual Cell models (VCell; http:// www.vcell.org) [37-41], we developed a geometry reduction algorithm preserved path reduction or PPR [36]. This algorithm simplifies the Purkinje neuron geometry to just the essential dendritic segments and branches in a path between a prototypical dendritic spine and the soma, while maintaining the dynamic electrical features of the full geometry in these respective compartments. The resulting model has only 17 compartments and allows us to readily couple the biochemistry in the spine compartment with the electrophysiology of the entire system. Reference [36] provides full details of the algorithm and the resultant geometry.

The baseline VCell model for normal physiology of the system was also previously published by our laboratory [35]. This compartmental model focused on IP3R1mediated calcium signaling in the Purkinje cell, taking into account the increased abundance and decreased 
sensitivity of the Purkinje cell IP3R1 relative to other cell types. The input to the system is provided as a train of IP3 pulses (representing IP3 production from multiple parallel fiber stimuli) with variable parameters that control the number, amplitude and duration of the IP3 pulses. In addition, the model included influx of calcium representing the opening of voltage-gated calcium channels when the Purkinje neuron is stimulated by a climbing fiber. It therefore allowed the study of coincident activation of the Purkinje spine by multiple parallel fibers and a single climbing fiber. The model also included the downstream effects leading to IP3R1-mediated calcium release, as well as calcium buffering and SERCA, which resides on the sER. All of the spine cytosol species are free to diffuse through the spine neck into the adjacent dendrite. IP3 also diffuses in the cytosol to bind its calcium channel-coupled receptor on the sER membrane. Calcium is released from sER stores into the cytoplasm and also co-activates IP3R1, as well as binds to various buffers. Some of this calcium is also pumped back into the sER by SERCA. The model definition in VCell includes specifying reactions, membrane transport mechanisms, stoichiometry, and kinetic rate expressions that correspond with these processes.

We subsequently created a 3D spatial model that explored [42] the membrane signaling mechanisms upstream of IP3R. We distinguished between candidate sources of sufficient phosphatidylinositol-4,5-bisphosphate (PIP2) in the spine for the production of requisite IP3. Local PIP2 sequestration provided an efficient means of fine-tuning coincident activation of parallel fiber and climbing fiber stimuli in cerebellar Purkinje neuron spines. The results reported in this study concluded that local high PIP2 achieved through a sequestration mechanism could provide sufficient IP3 for supralinear calcium release.

We used optimized parameters [42] to study physiological effects of varying IP3R1 sensitivity and abundance with a physiologically appropriate IP3 signal amplitude and duration. To manipulate IP3R1 abundance and sensitivity, we adjusted the parameters Jmax and $d_{\mathrm{IP} 3}$, respectively, in our comprehensive model (see a table of parameters in [42]). Small Jmax values represent reduced IP3R1 abundance. The default value is from Hernjak et al. [35], and is 10x larger than the IP3R1 determined for neuroblastoma cells, to account for the increased levels of IP3R1 in Purkinje neurons. The $d_{\text {IP3 }}$ parameter represents the dissociation constant of IP3 from IP3R1, so that high $d_{\text {IP3 }}$ values in our model represent low affinity of IP3R for IP3. We use the apparent affinity of IP3R1 for IP3 as a measure of sensitivity, as in the experimental study by Tang et al. [43]. Manipulation of these IP3R1parameters was done in the context of a train of 4 parallel fiber (PF) stimuli followed by a single climbing fiber (CF) stimulus, following the optimal protocol designed by Wang et al. [44]. This allowed us to examine the effects of IP3R1 insufficiency and supersensitivity in the context of Purkinje spine stimulation patterns similar to those in published experimental studies [35,42,44-46].

We investigate the pathophysiology of various SCAs, as well as the influence of IP3R1-mediated calcium release on the activity of voltage-gated $\mathrm{K}_{\mathrm{Ca}}$ channels, and thus on membrane potential. We also combine essential features of the spatial and compartmental models to create a $3 \mathrm{D}$ model of the spine. We use this model to probe the significance of Homer (associated with the postsynaptic density) physically linking mGluR to IP3R1. The resulting multicompartmental and $3 \mathrm{D}$ spatial Virtual Cell models can be accessed in the public domain at http://www.vcell.org under the shared username Brown. The models are:

1. "Brown et al. 2011 Analysis of SCA15-SCA16 with IC4 Peptide Application",

2. "Brown et al. 2011 SCA1 Compensation Analysis",

3. "Brown et al. 2011 Analysis of SCA1-SCA2-SCA3 with IC-G2736X Peptide",

4. "Brown et al. 2011 Purkinje Biochem-Electrophysiol SCA",

5. "Brown et al. 2011 Purkinje 3D Spine Spinocerebellar Ataxia study", and

6. "Brown et al. 2011 Combined Purkinje - Several Spines - Current Injection".

\section{Adapting the PPR model to the SCA model}

In order to investigate how biochemical signaling at the spine could affect electrophysiology, we needed to allow calcium release to influence the voltage-gated $\mathrm{K}_{\mathrm{Ca}}$ channels. In the PPR model, $\mathrm{K}_{\mathrm{Ca}}$ channels were only influenced by calcium influx into a phenomenological submembrane shell. This allowed for very high transient concentrations of calcium $(500 \mu \mathrm{M})$, as well as a high concentration of resting calcium $(\sim 4 \mu \mathrm{M})$ near the inner surface of the plasma membrane. Since such a high amount of basal calcium is not physiologically observed or observable (in the absence of Purkinje neuron stimulation by nerve endings from regulatory cells) and since calcium concentration beyond the submembrane shell was not explicitly considered in the PPR model, we felt it was important to model calcium less phenomenologically. Therefore, in this work, we removed submembrane shell calcium from the model only in the spine and the adjacent dendrite, where calcium signaling biochemistry is modeled explicitly. The adjacent dendrite was adjusted in tandem with the spine, because calcium and other molecules involved in biochemical processes are allowed to diffuse freely between the spine and the adjacent dendrite, restricted only by the geometry of the spine neck. In a previous 
study, Hernjak et al. [35] used a one-dimensional model to determine a dendritic distance, $28 \mu \mathrm{m}$ from a stimulated spine, at which biochemical concentrations remained at steady state during synaptic activity. The model therefore assumes that there is no net molecular flow between compartments, except for the adjacent dendrite (given a length of $28 \mu \mathrm{m}$ ) and the spine.

In other compartments, calcium influx feeds only into submembrane shell calcium. In the spine and adjacent dendrite, calcium influx is allowed to directly alter concentration of calcium involved in biochemical signaling; we term this calcium 'global calcium'. The concept of the submembrane shell in electrophysiological models [47-49] assumes a characteristic relaxation time of $2 \mathrm{~ms}$. This relaxation time represents the time it could take for submembrane shell calcium to relax to the 'global calcium' steady state, $40 \mathrm{nM}$ in the model. The submembrane shell relaxation time conceptually encompasses: (i) diffusion from beneath the membrane into the rest of the compartment, for example, the spine head, (ii) diffusion into adjacent compartments, for example, from the spine to the adjacent dendrite, (iii) buffering by calcium binding proteins, for example, calbindin and parvalbumin, (iv) uptake into the smooth endoplasmic reticulum by SERCA, and (v) extrusion through the plasma membrane by the $\mathrm{Na}-\mathrm{Ca}$ exchanger. In addition, calcium extrusion from the cell by the plasma membrane Ca-ATPase (PMCA, calcium pump) is modeled explicitly as a Michaelis-Menten expression.

In order to properly replace submembrane shell calcium with global calcium in the spine and the adjacent dendrite, (i) - (v) are all modeled explicitly. The model by Hernjak et al. [35] assumed instantaneous diffusion in the spine, and included diffusion between the spine and adjacent dendrite. It also included explicit buffering by calbindin and parvalbumin, in addition to calcium uptake by SERCA. That model did not include calcium extrusion from the spine or adjacent dendrite. Here, we add in calcium efflux by including the Michaelis-Menten Ca-ATPase from the Purkinje neuron electrophysiology models $[48,49]$ and a $\mathrm{Na}-\mathrm{Ca}$ exchanger from a study on cerebellar granule cells [50] (a similar $\mathrm{Na}-\mathrm{Ca}$ exchanger in a Purkinje neuron model was not available). The $\mathrm{Na}-\mathrm{Ca}$ exchanger is not electrogenic and simply provides an additional means of calcium extrusion; its kinetics are unchanged in our model. The total concentration of the Ca-ATPase is increased to allow for calcium efflux appropriate for maintaining a physiological steady state calcium concentration in the Purkinje neuron spine. The kinetics for the voltage-gated calcium-activated potassium channels were adjusted to be influenced by global calcium. This reflects results from experimental studies which suggest that BK channels can be activated by global calcium concentrations less than $10 \mu \mathrm{M}$ [51].

\section{The ICpeptide study}

The Bezprozvanny lab found that Protein Phosphatase 1 alpha $(\mathrm{PP} 1 \alpha)$ dephosphorylation of IP3R is largely dependent on PP1 $\alpha$ binding the very tip of the C-terminal of IP3R1, and that IP3R1 dephosphorylation (increased IP3R1-PP1 $\alpha$ binding) decreased the receptor's sensitivity to IP3 $[21,43]$. Binding of PP1 $\alpha$ to the tip of the endogenous IP3R1 C-terminal and to peptides that resemble the C-terminal of IP3R1 (Figure 2) (here termed ICpeptides, e.g., GST-tagged IC4 in Figure 1a) was added to the model at the ER membrane and in the cytosol, respectively, with mass action kinetics and a stoichiometry of 1 . For example:

$$
K_{f_{-} I C 4}{ }^{*} P P 1 \alpha^{*} I C 4-K_{r_{-} I C 4}{ }^{*} I C 4_{-} P P 1 \alpha
$$

where $K_{f_{-} I C 4}$ and $K_{r_{-} I C 4}$ are the forward and reverse rate constants, respectively, for $P P 1 \alpha$ binding the exogenous peptide IC4, and IC4_PP1 $\alpha$ represents PP1 $\alpha$ bound to the IC4 peptide;

$$
K_{f_{-} I P 3 R 1_{-} C t}{ }^{*} P P 1 \alpha^{*} I P 3 R 1_{-} C t-K_{r_{-} I P 3 R 11_{-} C t}{ }^{*} I P 3 R 1-C t_{-} P P 1 \alpha
$$

where $K_{f_{-} I P 3 R 1_{-} C t}$ and $K_{r_{-} I P 3 R 1_{-} C t}$ represent the forward and reverse rate constants for PP1 $\alpha$ binding the endogenous tip of the IP3R1 C-terminal, and IP3R1_Ct_PP1 represents PP1 $\alpha$ bound to IP3R1 at the smooth ER (sER). Rate constants are provided in Table 2. It was assumed that PP $1 \alpha$ bound the ICpeptides and the endogenous IP3R1 with the same affinity.

The C-terminal of IP3R1 can also bind mutant Ataxin. The affinity of the IP3R1 C-terminal tip was taken to be greater for the mutant protein than for PP1 $\alpha$. This was done to incorporate two phenomena into the binding reaction. First, planar lipid bilayer reconstitution experiments from Chen et al. and Liu et al. $[6,10]$ suggest that direct association of the mutant ataxin protein with the C terminal of IP3R1 leads to supersensitivity of the receptor. Second, the binding domain of the mutant protein [52] on IP3R1 overlaps the PP1 $\alpha$ binding motif [52] on IP3R1. Mutant protein binding IP3R1 in situ (as in cerebellar slices from mouse models that can be used to test simulation predictions; see Discussion) may decrease PP1 $\alpha$ interaction with the receptor. These two factors are effectively modeled by assigning a larger affinity for mutant protein binding to IP3R1 than PP1a binding the receptor. Affinities were captured by the rate constants, all available in Table 2.

\section{Results}

Two main categories of ataxias in humans and mouse models are considered in this study: those with reduced IP3R1 and those with supersensitive IP3R1 (Table 1). However, some ataxias involve both phenomena, adding 


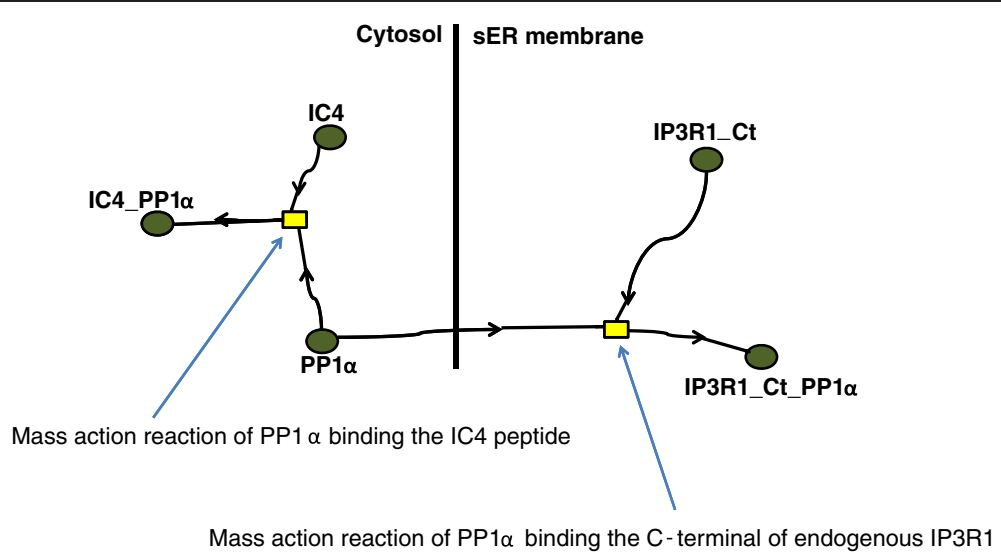

Figure 2 Schematic for PP1a binding endogenous IP3R1 and a peptide resembling the C-terminal of IP3R1 (IC4). This corresponds to the ICpeptide study described in the Methods section.

a layer of complexity. Further, a common component of all these ataxias is abnormal calcium metabolism.

\section{SCA15/16: pathophysiology of IP3R1 insufficiency} In ataxias with reduced IP3R1, e.g., SCA15/16, receptor levels appear to be decreased by varying amounts, even in conditions of heterozygous gene deletion [3,4,14$16,20]$. We captured this variability in our model by adjusting the abundance parameter Jmax to decrease IP3R1 levels to $50 \%, 40 \%, 30 \%, 25 \%$, and $10 \%$ of the original value. Figure $3 \mathrm{a}$ shows the supralinear calcium response with simulated wild type IP3R1 levels (labeled as 'Original IP3R1 density') when the Purkinje neuron spine is stimulated by a single climbing fiber at $0.15 \mathrm{~s}$ (labeled as 'Ca2+ influx only'), by 4 pulses from a single parallel fiber at $0.1 \mathrm{~s}$ (labeled as 'Ca2 release only'), or by coincident stimulation by 4 pulses from a parallel fiber at $0.1 \mathrm{~s}$ and a climbing fiber at $0.15 \mathrm{~s}$. The simulation uses Magnesium Green as the calcium indicator $\left(K_{d}=19 \mu \mathrm{m}\right)$ that fluoresces more brightly when bound to calcium $[35,44]$. The figure indicates that the coincident calcium transient is greater than the sum of the individual calcium transients from stimulation of only the climbing fiber or only the parallel fiber $[35,42,54]$; in our simulations, calcium release alone produces a barely perceptible increase in free $\mathrm{Ca}^{2+}$, but when it occurs together with calcium influx, the free $\mathrm{Ca}^{2+}$ increase more than doubles compared to influx alone. The figure also shows that halving the density of IP3R1 (as in heterozygous mouse models and individuals) severely reduces supralinear calcium release. Thus, the model can be used to investigate the heterozygous human ataxia SCA15/16.

Table 2 Parameters for new features added to model

\begin{tabular}{|c|c|c|c|}
\hline Model Parameter & Value & Comment & Reference \\
\hline $\mathrm{K}_{\mathrm{f}-\mathrm{IC} 4}$ & $0.001 \mathrm{uM}-1 . \mathrm{s}-1$ & Forward rate of IC4 binding PP1a & - \\
\hline $\mathrm{K}_{\mathrm{r}-\mathrm{IC} 4}$ & $0.0005 s-1$ & Reverse rate of IC4 binding PP1a & - \\
\hline$K_{f-1 P 3 R 1-C t}$ & $10 \mathrm{uM}-1 . \mathrm{s}-1$ & Forward rate of IP3R1 C-terminal binding PP1a & - \\
\hline $\mathrm{K}_{\mathrm{r}-\mathrm{P} 3 \mathrm{P} 1-\mathrm{Ct}}$ & $0.0005 s-1$ & Reverse rate of IP3R1 C-terminal binding PP1a & - \\
\hline$K_{\text {f-mutant-IC4 }}$ & $20 \mathrm{uM}-1 . \mathrm{s}-1$ & Forward rate of IC4 binding mAtaxin & - \\
\hline $\mathrm{K}_{\mathrm{r}-\text { mutant-IC4 }}$ & $0.0005 s-1$ & Reverse rate of IC4 binding mAtaxin & - \\
\hline$K_{f-m u t a n t-P 3 R 1-C t}$ & $20 \mathrm{uM}-1 . \mathrm{s}-1$ & Forward rate of IP3R1 C-terminal binding mAtaxin & - \\
\hline $\mathrm{K}_{\mathrm{r}-\text { mutant-IP3R1-Ct }}$ & $0.0005 s-1$ & Reverse rate of IP3R1 C-terminal binding mAtaxin & - \\
\hline mAtaxin_init & variable & Mutant Ataxin PolyQ Protein & - \\
\hline PP1a_init & $1 \mathrm{uM}$ & Directly binds tip of IP3R1 C-terminal & {$[43]$} \\
\hline IP3R1-Ct_init & $1 \mathrm{uM}$ & Tip of IP3R1 C-terminal & - \\
\hline IC4_init & $2 \mathrm{uM}$ & Competitively inhibits PP1a binding & [43] \\
\hline IC-G2736X_init & $2 \mathrm{uM}$ & Associates with polyQ region & {$[52]$} \\
\hline IC10_init & variable & Competitively inhibits polyQ binding & [53] \\
\hline
\end{tabular}



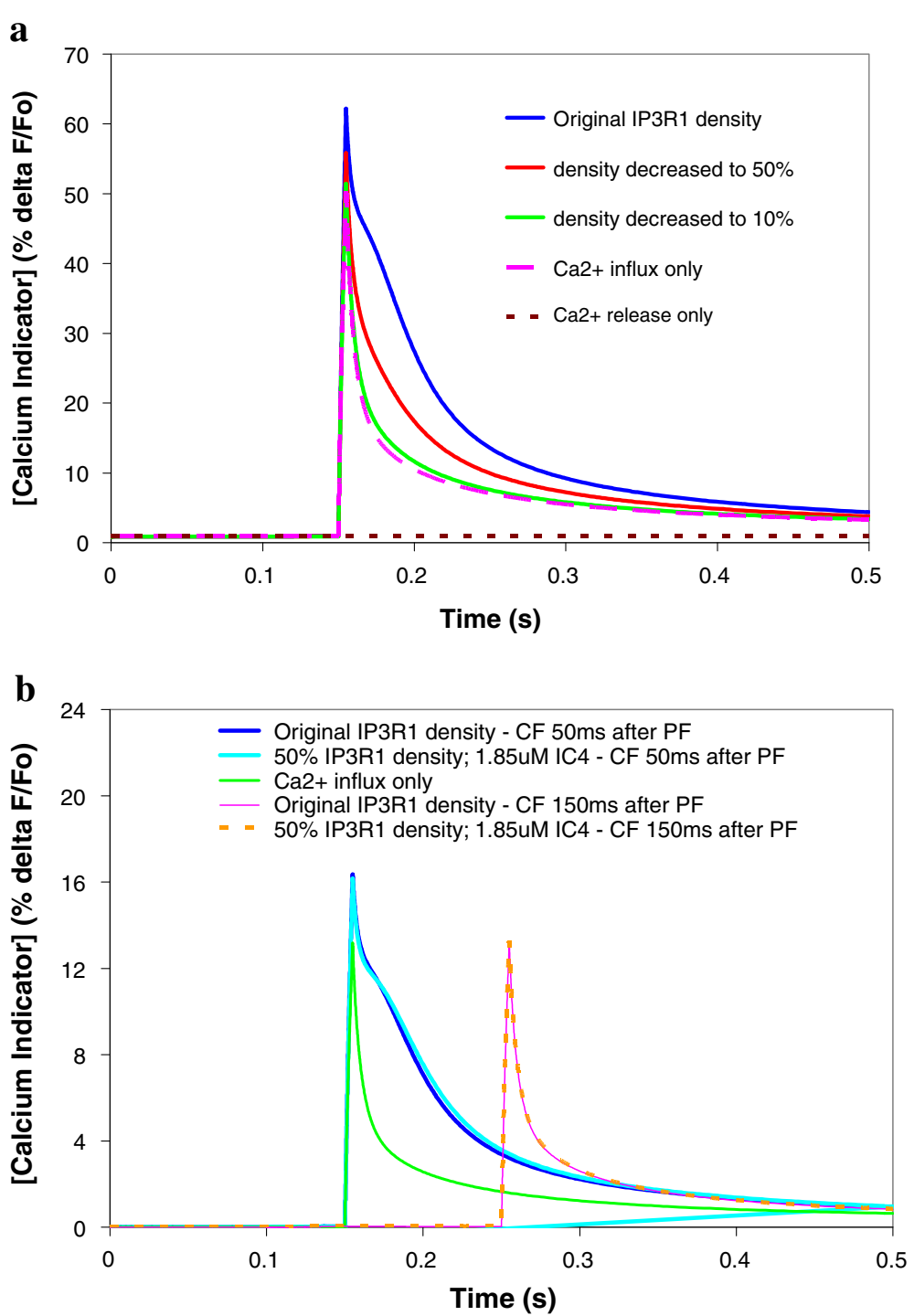

Figure 3 Increasing IP3R1 sensitivity to IP3 can restore normal calcium response in the context of reduced IP3R1 levels (a model of SCA15/16). a, Coincident calcium release (from 4 parallel fiber stimuli and a single climbing fiber stimulus) with IP3R1 levels decreased to 50\% and 10\%. b. Wild type levels of supralinear calcium release can be regained in IP3R1 insufficiency by augmenting IP3R1 sensitivity to activation by IP3, using IC4 at a concentration that gives a 1.7-fold increase in IP3R 1 sensitivity to IP3. This fold increase in sensitivity is within an experimentally observed range (2-fold) [55]. Fine-tuning of coincidence detection [42] is preserved.

\section{Application of ICpeptide to adjust sensitivity in SCA15/16} Modeling results from Hernjak et al. [35] showed that increasing the sensitivity of the IP3R 1 by a factor of 10 did not compensate for a decreased the level of IP3R1 to $10 \%$ of the wild type value. They argued that the normally low sensitivity of IP3R1 to IP3 was required to assure that calcium release is confined to the activated dendritic spine while the normally high level of IP3R assures robust calcium release despite the low sensitivity. This result is consistent with the finding in Figure 3a, which shows that the calcium transient with $10 \%$ IP3R1 abundance is almost entirely due to calcium influx through voltage-gated calcium channels. Very little of the calcium transient is due to calcium release from the ER, through IP3R. It allows us to suggest that in Purkinje cells, with have much lower sensitivity of IP3R to IP3 than in other cell types, decreasing receptor density to $10 \%$ of the wild type levels (which amounts to replacing Purkinje neuron IP3R with levels found in other cell types) is as good as having no IP3R at all. Figure 4 shows that increasing sensitivity in the wild type context (by adding mutant Ataxin-2 to the model with normal IP3R levels) disrupts the IP3 response [35]. However, in the context of decreased Purkinje IP3R abundance, augmented sensitivity (whether comparable to other cell types or greater) could potentially restore normal IP3 response if the IP3R1 is not lowered as 


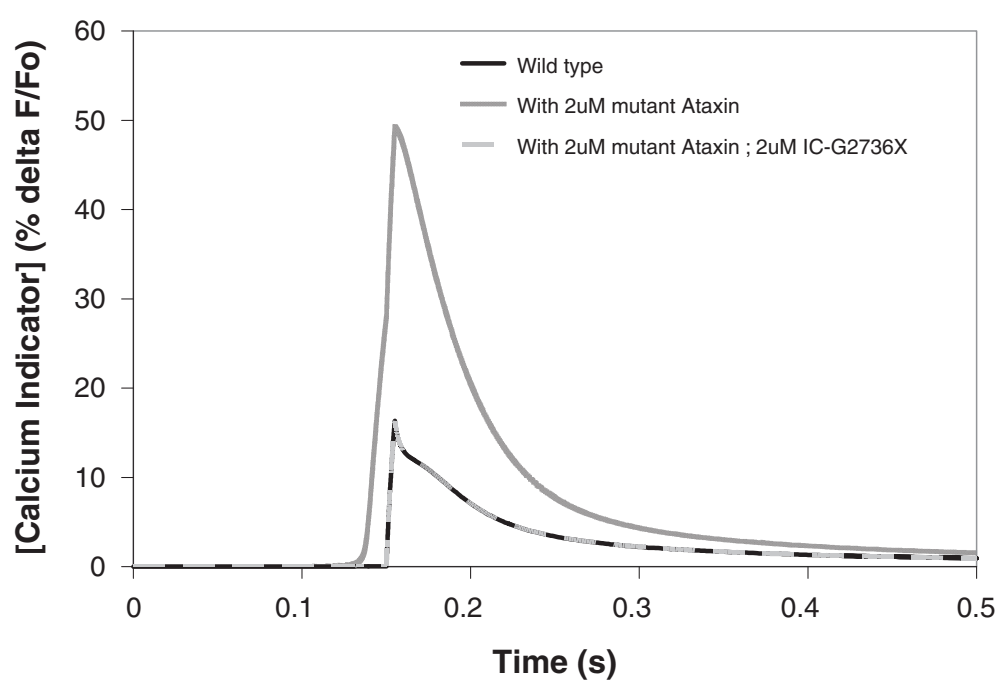

Figure 4 Simulated application of another ICpeptide in a model of SCA1/2/3 can restore normal calcium release. Hyperactive calcium release in the presence of mutant Ataxin can be overcome by application of IC-G2736X, which resembles the polyglutamine binding domain in the C-terminal of IP3R1, and was developed by Tang et al. [52]. Note that the dashed light grey line (representing restoration of normal calcium release by application of ICpeptide in the context of a mutant Ataxin model) directly superimposes on the solid black line (which represents wild type).

drastically as the factor of 10 used in the Hernjak [35,54] model.

To assess this, we simulated an additional system (see Table 2) that has been shown experimentally to regulate IP3R1 sensitivity: the phosphorylation state of IP3R1. Tang et al. discovered that dephosphorylation of wild type IP3R1 by $\mathrm{PP} 1 \alpha$ profoundly reduced the receptor's sensitivity to IP3 and decreased IP3R1-mediated calcium release [43]. Indirect binding occurs through AKAP, an adaptor protein that links IP3R1 to both PKA and PP1 $\alpha$ pha by binding to the LIZ domain in IP3R1 (see Figure 1a). Tu et al. showed that most association of $\mathrm{PP} 1 \alpha$, the major dephosphorylator of IP3R1, to the receptor occurs via direct binding at the C-terminal of IP3R1 [21] (see Figure 1a). Tang et al. created GST-IC4, a competitive peptide that resembles the tip of the C-terminal of IP3R1 (36 aa, Q2714-A2749) [43] (Table 3). This peptide binds PP1 $\alpha$, preventing its direct association to the C-terminal of IP3R1 (see Figure 1a). They found that dephosphorylation of IP3R1 could be reduced by more than twofold by administering GST-IC4. In the model, we simulated treating Purkinje neurons of SCA15/16 mice with GST-IC4 to see if the resulting increase in IP3R1 sensitivity to IP3, could augment IP3R1mediated calcium release. Figure $3 \mathrm{~b}$ shows that as IC4 increases, the resulting decreased association of PP1 $\alpha$ with IP3R1 increases the sensitivity of the receptor for IP3 and restores normal calcium transients. In the figure, a 1.7-fold increase in IP3R1 sensitivity corresponds to $1.85 \mathrm{uM}$ of IC4 needed to restore normal calcium response when IP3R1 levels are decreased by $50 \%$. This suggests that an experimental study of GST-IC4 treatment in mouse models of SCA15/16 could show normalized IP3 response with a fold increase in sensitivity already demonstrated by Tang et al. in in vitro studies [43]. However, as levels of IP3R1 go lower and lower, the fold increase in sensitivity needed to restore normalcy increases nonlinearly (data not shown). In the model, IP3R1 levels could be decreased only as far as to $25 \%$ and still be able to restore normal calcium with a fold increase in sensitivity that is experimentally attainable.

The simulations were repeated with the climbing fiber stimulus applied $150 \mathrm{~ms}$ after the start of the train of the PF stimuli, unlike the previous results that have a timing difference of $50 \mathrm{~ms}$. A $150 \mathrm{~ms}$ timing between the CF

Table 3 Suggested use of ICpeptides relevant to this study

\begin{tabular}{|c|c|c|c|c|}
\hline ICpeptide & Base pairs & Minimal binding region of target & Use suggested by this study & Reference \\
\hline \multirow[t]{2}{*}{ IC4 } & Q2714-A2749 & R2731-G2736 & in vivo competitive inhibition of PP1a & (Tang et al., 2003b, \\
\hline & & & with reduced levels of IP3R1 & Tu et al., 2004) \\
\hline \multirow[t]{2}{*}{ IC-G2736X } & D2590-G2736 & F2627-G2736 & in vitro/vivo competitive inhibition of mutant Ataxin & (Tang et al., 2009) \\
\hline & & & with supersensitive IP3R1 & \\
\hline IC10 & F2627-A2749 & F2627-G2736 & possible alternative to IC-G2726X & (Tang et al., 2009) \\
\hline
\end{tabular}


and PF stimuli falls outside the optimal time window suggested by both experiments [44,56] and modeling [42]. Figure $3 \mathrm{~b}$ shows that with this $150 \mathrm{~ms}$ time difference, supralinear calcium release is lost in the wild type case, as well as in the heterozygous case. This suggests that restoration of normal calcium release in the model does not alter fine-tuning of coincidence detection suggested by Brown et al. [42]. Thus, increasing IP3R1 sensitivity could be explored as a therapeutic tactic in SCA15/16 mouse models, e.g. IP3R1 $1^{\text {delta18/delta18 }}$ or $\mathrm{IP} \mathrm{R} 1^{+/-}$mice.

\section{SCA2: application of ICpeptide in ataxias with pathologically increased sensitivity}

Tang et al. also found that the huntingtin binding site on IP3R1 is immediately upstream of the PP1 $\alpha$-binding motif on the tip of the IP3R1 C-terminal [52]. The predicted minimal region on IP3R1 for huntingtin polyglutamine repeat binding (F2627-G2736) actually overlaps a small portion (5 aa) of the minimal PP1-binding motif (R2731-A2749)[52]. The huntingtin protein binding IP3R1 may therefore physically occlude or partially hinder binding by PP1 $\alpha$ lpha in vivo and in situ. This would reduce PP1 $\alpha$ lpha dephosphorylation of the receptor, leaving it in a hyperphosphorylated and therefore supersensitive state. A similar mechanism should occur with mutant Ataxins in SCA2 [10] and SCA3 [6] and also SCA1 $[8,10,57]$, given that the mutant Ataxin and huntingtin proteins all have similarly expanded CAG repeats.

Figure 4 shows that adding $2 \mu \mathrm{M}$ mutant Ataxin, e.g. mutant Ataxin-2 in SCA2, to the wild type model triples the increase in simulated fluorescence of the calcium indicator. Furthermore, the IP3R1 supersensitivity allows calcium concentration to start rising even before coincidence with calcium influx. In addition, we simulated the application of a different ICpeptide in our model. This ICpeptide, IC-G2736X (D2590-G2736)(Table 3), was also created by Tang et al., and encompasses the part of IP3R1 that encodes the minimal binding region for the expanded polyglutamine repeats in huntingtin, and likely the mutant ataxin proteins [52]. Further, Tang et al. used a $\beta$-galactosidase assay to show that IC-G2736X strongly associates with the mutant polyQ huntingtin [52]. In fact, they also used viral infection to insert IC10 into striatum of ataxic HD mice, and observed consequent stabilized calcium signaling, as well as neuroprotective effects [53]. In our model, IC-G2736X competitively binds the mutant Ataxin protein and blocks mAtaxin from binding endogenous IP3R1. The normal sensitivity of IP3R1 to activation by IP3 is therefore restored, and IP3R1 is no longer supersensitive. Figure 4 shows the restoration of normal supralinear calcium release. This result allows us to predict that experimental application of such an ICpeptide in cerebellar slices from mice with ataxias associated with supersensitive IP3R1 and increased calcium release may similarly restore normal calcium response. We expect that various concentrations of ICpeptide could be needed to normalize IP3R1 sensitivity and consequently calcium release in SCA1, SCA2, or SCA3, based on the degrees to which IP3R1 sensitivity to IP3 is pathologically increased. This could depend on the concentration of the mutant protein in the spine, as well as other unknown factors.

\section{SCA1 (\& SCA3): compensation versus pathology}

Although expression of IP3R1 and other calcium signaling proteins is reduced in SCA3 mice [7] and in SCA1 mice and humans $[9,11]$, reduced calcium release is not experimentally observed in either disease. It is therefore not understood what role reduced IP3R1 plays in the pathology of these polyQ diseases. Figure 3 suggested that in SCA15/16 and other ataxias with reduced IP3R1 abundance and consequently reduced calcium release, therapeutic strategies to increase IP3R1 sensitivity to activation by IP3 could restore normal calcium release. This begs the question of whether, conversely, the downregulation of IP3R1 in ataxias with supersensitive IP3R1 could partially or wholly restore normal calcium response. A glimpse into a compensatory effect could lie in examining actual experimentally observed relative expression levels in SCA1 $[9,11]$, and using those observed levels in the model with supersensitive IP3R1. This allows the modeling and simulations to be driven by experimental observations. Accordingly, a 1.8-fold decrease (approximated from Lin et al. and Serra et al.) $[9,11]$ was simulated in the model for SCA1 pathology.

Figures $4 \mathrm{a}$ and $4 \mathrm{~b}$ show that tuning down IP3R1 decreases the hyperactive supralinear calcium release to levels closer to wild type. This suggests that experimentally observed reduced abundance of IP3R1 may partially compensate for supersensitivity in SCA1 mice. However, other calcium signaling molecules were also downregulated by at least 1.8 -fold $[9,11]$. To determine the individual effect of the downregulation of some of the molecules, each molecule was sequentially downregulated. Tuning down SERCA in addition to IP3R1 has no additive effect. Myosin Va (MyoVa) and Homer 3 also are decreased $[9,11]$. MyoVa and Homer 3 are thought to potentially (i) guide sER into the spine, and (ii) anchor SER in the spine, respectively [58,59]. Decreasing sER volume in the spine by 1.8 -fold in the ataxia model reduces the hyperactive calcium release to levels similar to tuning down IP3R1 or IP3R1 and SERCA. Although the correlation between Homer and MyoVa expression and sER volume fraction is probably not linear, it is remarkable that reducing the volume fraction by 1.8 -fold gives a result that is close to directly tuning down IP3R1 
and SERCA, which reside on the sER. Given the critical importance of IP3R1 on the sER in normal Purkinje neuron function, reduced volume fraction of spine sER could contribute to the pathophysiology of IP3R1associated ataxia. However, in the context of IP3R1 supersensitivity, Homer and MyoVa downregulation could be compensatory. Finally, tuning down mGluR (by tuning down pulsatile IP3 production) in addition to SERCA and IP3R1 further decreases hyperactive calcium release, so that the resulting supralinear calcium release in the ataxic model is even closer to wild type. This is remarkable, suggesting that mGluR downregulation $[9,11]$ is also compensatory, and additive to IP3R1 downregulation. Not surprisingly, Figure $5 \mathrm{~b}$ suggests that the greater the concentration of mutant Ataxin, the less the compensation achieved by tuning down IP3R1, SERCA, mGluR, and Homer.

Expression of calcium-binding proteins, parvalbumin and calbindin, are also experimentally observed to be reduced $[9,11]$. It is not known whether this contributes to pathology or compensation. Calbindin knockout mice crossed with SCA1 mice worsens the phenotype [9]. This suggests that low levels of these buffer proteins could accelerate pathology. It is possible that normal or high levels of buffer proteins may help to maintain homeostasis in the presence of supersensitive IP3R1 early on in the SCA1 disease, then, when their expression is reduced, homeostasis is lost. Modeling results support this suggestion. Tuning down the calcium buffers parvalbumin and calbindin in the model makes supralinear calcium more robust than with down regulation of IP3R1, mGluR and SERCA alone (Figure 5a,b). Accordingly, the downward trend in the peak concentration of calcium is reversed with down regulation of calcium buffers. This is consistent with studies in Purkinje cells from parvalbumin and calbindin knockout mice that are ataxic [60,61], where peak amplitudes of calcium transients evoked by climbing fiber stimulation were double those in wild type mice [62]. Notably, it has been suggested that because reduced expression of buffer proteins is observed in SCA1 mice
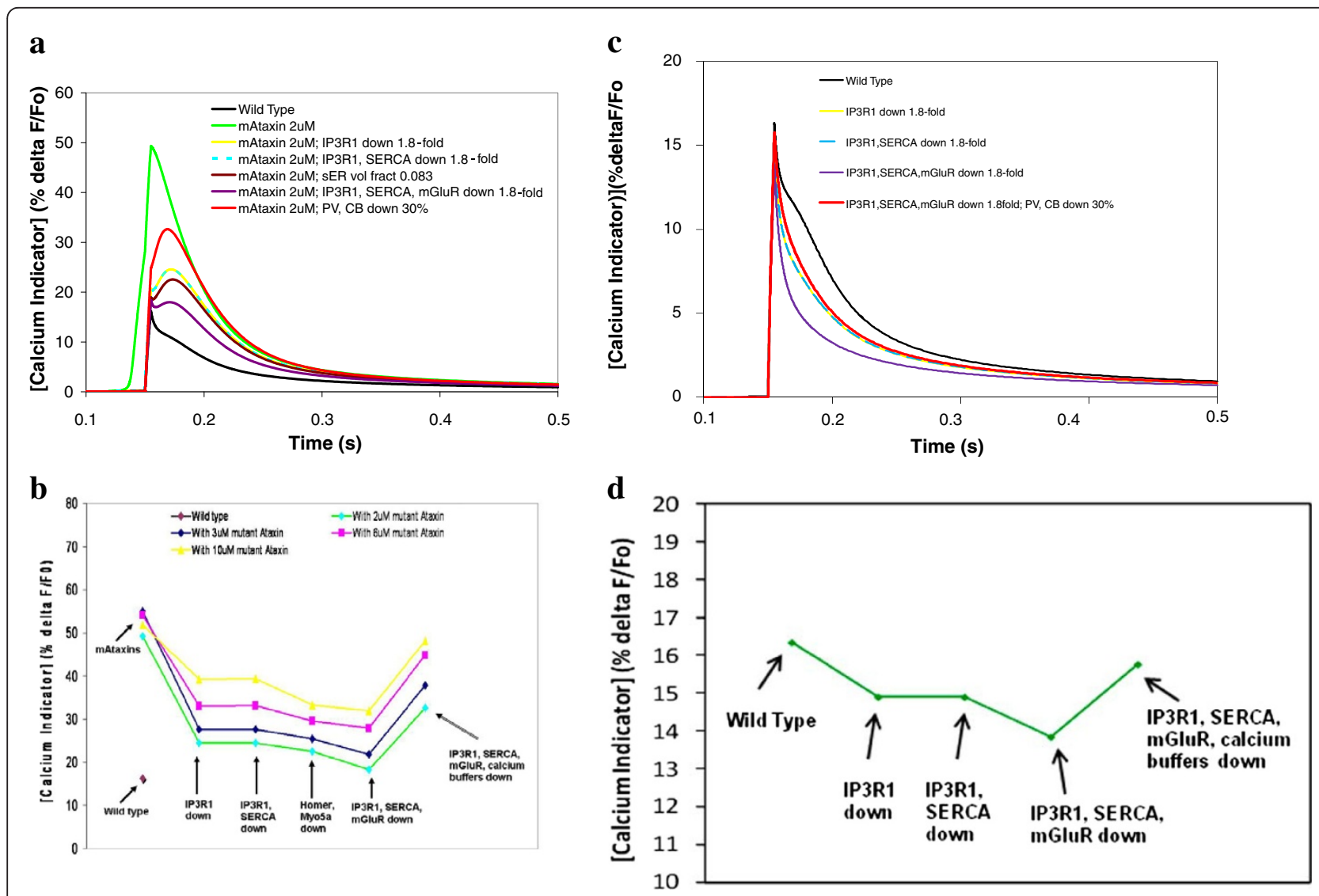

Figure 5 Downregulation of IP3R1, SERCA, mGluR, and Homer/MyoVa appears to be compensatory, but downregulation of calcium buffers may contribute to pathology. a \& c, Supralinear calcium release in wild type and with mutant Ataxin and with each molecule downregulated cumulatively, assuming supersensitive IP3R1 (a) or normosensitive IP3R1 (c). For the red curve, the calcium buffers PV and CB are downregulated by $30 \%$ in addition to at least a 1.8-fold decrease in concentration for IP3R1, SERCA, and mGluR. $\mathbf{b} \& \mathbf{d}$, The compensation trend plotted for various potential concentrations of mAtaxin, showing the inverse relationship between the extent of compensation and the relative concentration of mAtaxin in the spine, assuming supersensitive IP3R1 (b) or normosensitive IP3R1 (d). 
before onset of symptoms, the timing of buffer protein downregulation may help determine the onset of symptoms $[63,64]$.

Notably, Liu et al. report that mutant SCA1 pathologically associates with IP3R1 [10], as do SCA2 and SCA3 $[6,10]$, suggesting a common supersensitive IP3R1 pathology, but they do not actually measure or report changes in IP3R1 sensitivity. Thus, we thought it was important to consider changes in calcium resulting from signaling protein downregulation, even if hyperactive IP3R1 is not assumed. Figures 5c and 5d illustrate that calcium responses resulting from IP3R1 and other calcium signaling protein downregulation, but with normal IP3R1 sensitivity, are less robust than wild type. This indicates that downregulation of these proteins in the absence IP3R1 supersensitivity does not appear consistent with the elevated calcium response relative to wild type, as observed in SCA1 mice [8]. The model therefore supports the hypothesis of IP3R1 supersensitivity in SCA1.

\section{Influence of calcium on voltage-gated calcium-activated potassium channels}

To better understand the interplay between calcium signaling and electrophysiology, we investigated the relationships between calcium influx, IP3R1-induced calcium release, and the voltage-gated calcium-activated potassium $\left(\mathrm{K}_{\mathrm{Ca}}\right)$ channels. Our model includes the BK channel ('big' conductance calcium-activated voltagegated potassium channel) and IK (intermediate conductance calcium-activated voltage-gated potassium channel) channels. Since more is known about the BK channels, we focused our study design and interpretation on these channels (see schematic in Figure 1b). The BK channel in the cerebellum is thought to contribute to repolarization of membrane potential transients in the dendrites, particularly calcium spikes (transient depolarization of membrane potential in spines and dendrites) resulting from the opening of voltage-dependent calcium channels in the dendrites [49]. In addition, experimental results by Sausbier et al. using ataxic BK knockout mice suggest that these channels are also important for the afterhyperpolarization of action potentials at the soma [65]. BK channels contribute to precision timing, and are thus important for normal firing of the cerebellar Purkinje neurons [65-70].

IP3R1 interacts closely with the BK channel, in glioma cells [71]. The BK channels seem to be in lipid rafts in the plasma membrane apposed to the sER. It is thought that in other cell types, including some neurons, the BK channels may form physical complexes with various plasma membrane calcium channels, bringing them within a few nanometers of the calcium channel pores [72]. We suspect that in the cerebellar Purkinje neuron spines, BK activity is also closely linked with the IP3R1-mediated calcium release. This idea is supported by direct activation of the BK channel by IP3R1-mediated calcium release in pyramidal neurons [73] and cereberal artery smooth muscle cells [74]. We therefore set out to investigate possible activation of BK channels (and IK channels) by IP3R1mediated calcium release.

In the model, we allowed calcium released from the sER in eleven spines to activate the voltage-gated $K_{\mathrm{Ca}}$ channels. A group of eleven spines represents a small portion of a dendrite branchlet. (The Purkinje neuron has $\sim 14$ spines per $\mu^{2}$ (Harris and Stevens, 1988)). Figure $6 \mathrm{a}$ shows that a $20 \mathrm{nA}$ current injected at the soma for $400 \mathrm{~ms}$ gives a single sodium action potential spike, followed by a gradual incline. When calcium influx due to CF stimulation is applied $40 \mathrm{~ms}$ after the start of the current injection, an identical result is obtained (data not shown). When calcium release due to PF stimulation is applied $20 \mathrm{~ms}$ before the start of the current injection, a single sodium action potential spike is obtained, followed by the gradual incline, but then terminates with a short action potential train (Figure 6b). These action potential oscillations are similar to tonic firing oscillations observed experimentally [65]. An identical result is obtained when calcium influx is also added in $40 \mathrm{~ms}$ after the start of current injection. Thus in contrast to Figures 2 and 3, where calcium release is stimulated in only one spine, the transient calcium influx may not significantly enhance the overall effect of calcium release in 11 spines. This point is corroborated by examination of the corresponding calcium transients shown in Figure 6c. With PF stimuli only, in the absence of current injection, the Ca transient is minimal. With current injection at the soma, in the absence of biochemical signaling, there is a significant calcium transient at the level of the spines, corresponding to that first membrane potential spike. During that spike, sodium channels open at the soma, and calcium channels open throughout the dendrite. That allows calcium to rush into the cell. The calcium concentration then gradually rises, corresponding to the membrane potential incline. With PF stimuli preceding current injection onset by $20 \mathrm{~ms}$, IP3 is already bound to IP3R1 when calcium rushes in through voltage-gated channels in the spines (in response to the depolarization of the membrane during current injection). This calcium also binds to IP3R1 on the sER. Thus, we have IP3 and calcium bound to IP3R1, yielding coincident activation of the receptor. In this case, coincidence detection is not of PF and CF stimuli, but of PF stimuli and current injection. IP3R1 can therefore serve as a coincidence detector for any activation of the Purkinje neuron that leads to changes in intracellular calcium concentration and increases in IP3 concentration. The figure shows that the resulting calcium transient is supralinear. With the CF stimuli only, a sharp calcium transient is obtained. When 


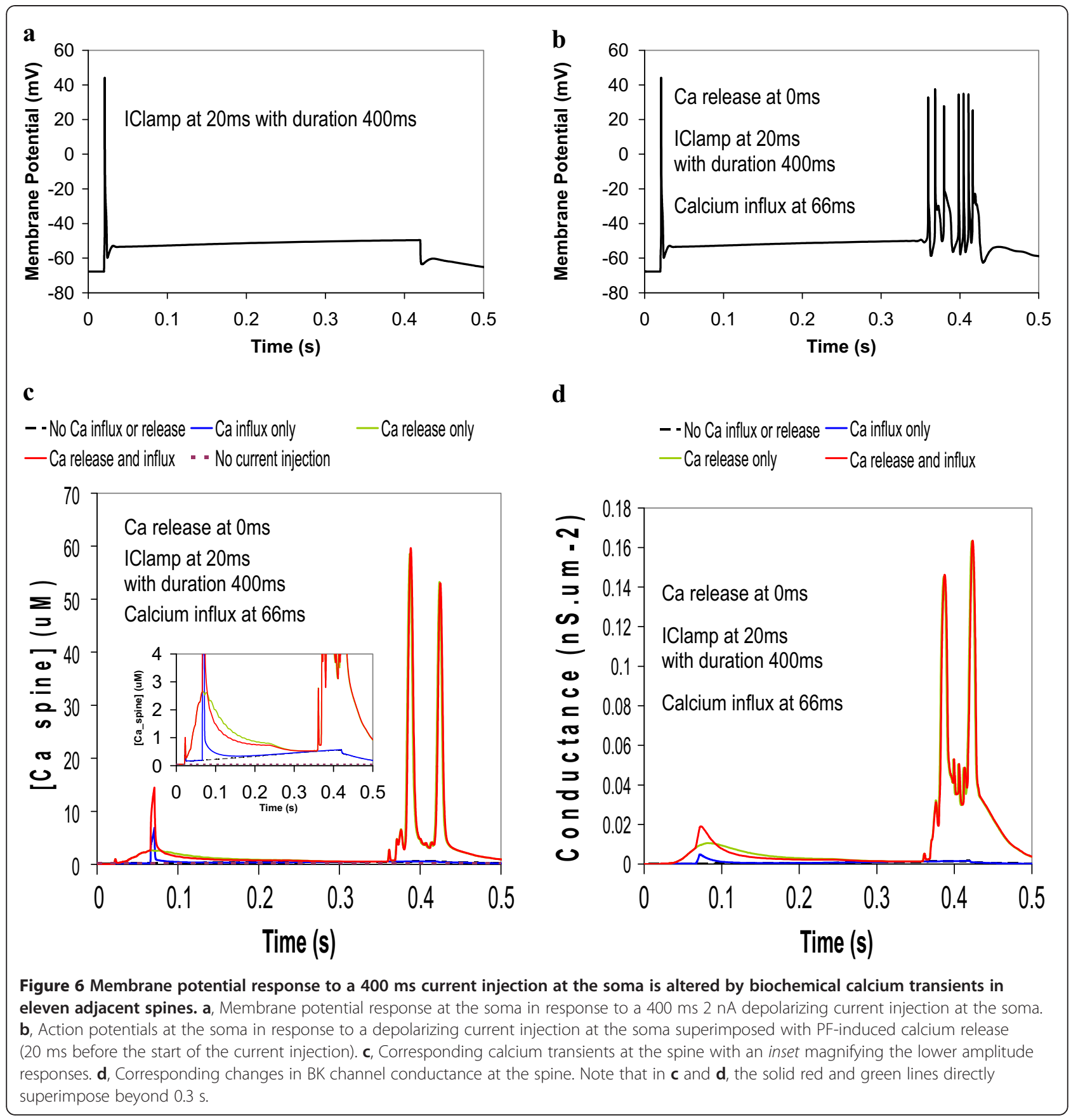

the CF stimulus is combined with current injection, the calcium transient is simply additive. This is because neither CF stimulus nor current injection provides IP3, which is necessary for coincidence detection at IP3R1. The corresponding modulation in the conductance of the BK channels is shown in Figure $6 \mathrm{~d}$. The figure indicates that BK channel conductance begins to rise almost immediately after either calcium release or calcium influx or both. Not surprisingly, the supralinear calcium transients give the biggest increases in conductance of the BK channels. The influence of the calcium transients on BK channels alters the membrane potential of the Purkinje neuron. The figure shows that with the calcium release from PF stimulus only, termination of the current injection results in a short train of action potentials. As with the membrane potential in Figure 6a, this spike does not occur in the absence of the PF stimulus. With supralinear calcium release activating BK channels, this also gives a termination spike that is identical to that obtained with calcium release only. CF stimulus alone does not alter the membrane potential.

In Figure 7, current injection commences significantly before both the PF and CF stimuli: PF stimulus occurs 

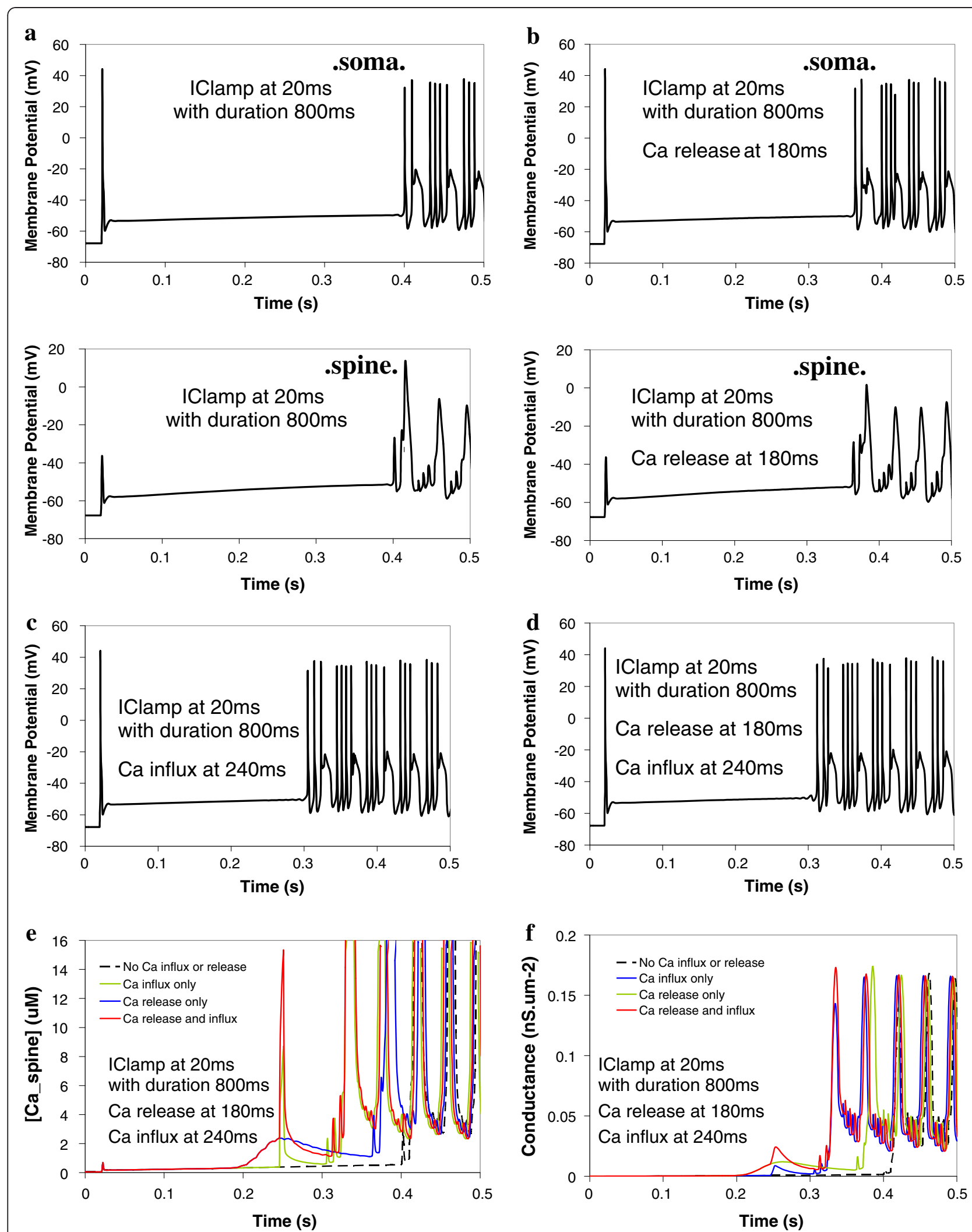

Figure 7 (See legend on next page.) 
(See figure on previous page.)

Figure 7 The onset of action potential oscillations is altered by calcium transients in eleven adjacent spines, with an 800 ms current injection and a different timing sequence between PF and CF stimuli. a, Membrane potential oscillations at the soma (top panel) and the spine (bottom panel) in response to a $800 \mathrm{~ms}$ depolarizing current injection at the soma (no calcium influx or release). b, Membrane potential oscillations at the soma (top panel) and at the spine (bottom panel) in response to a depolarizing current injection at the soma superimposed with calcium release only (160 ms after the start of the current injection). c, Membrane potential oscillations at the soma in response to a depolarizing current injection at the soma superimposed with calcium influx only (220 ms after the start of the current injection). d, Membrane potential oscillations at the soma in response to a depolarizing current injection at the soma superimposed with both calcium release and influx. e, Corresponding calcium transients at the spine. f, Corresponding changes in BK channel conductance.

$160 \mathrm{~ms}$ after current injection, but still occurs $60 \mathrm{~ms}$ (within the experimentally observed time window [56]) before the CF stimulus. In addition, the current injection now lasts for $800 \mathrm{~ms}$, as opposed to $400 \mathrm{~ms}$ in Figure 6. Figure $7 \mathrm{a}$ shows that in the presence of a prolonged current injection, a train of action potentials can be induced in the absence of any calcium increase. Consistent with Figure 6, with PF stimulus only, action potential spikes at the soma appear earlier (Figure $7 \mathrm{~b}$, top panel) than in the absence of the resulting biochemical calcium transient (Figure 7a, top panel). The bottom panels in Figures $6 \mathrm{a}$ and $6 \mathrm{~b}$ show the corresponding calcium potential spikes at the spine, with similar results. These calcium spikes occur once a depolarization threshold is reached in the dendrites and spines, in response to high frequency repetitive firing of the Purkinje neuron (corresponding to high amplitude current injection) [49]. The resulting change in membrane potential in the dendrites and the spines propagates to and is reflected at the soma as the transient plateaus that occur in the midst of the sodium spikes. The resulting calcium transients are similar to those in Figure 6, in that superposition of parallel fiber stimulus and current injection gives supralinear calcium release, and further superposition with CF stimulus gives a calcium transient that is additive (Figure 7e). Also similarly, the supralinear calcium transient from calcium release and current injection leads to earlier calcium signal oscillations than in the absence of the biochemical calcium signal (Figure 7e). Interestingly, the calcium obtained from CF stimulus superimposed with current injection results in earlier membrane potential spiking (Figure 7c) than the supralinear calcium release from PF stimulus and current injection superposition (Figure 7b). Virtually identical results are obtained with coincident $\mathrm{CF}$ and PF activation superimposed with current injection (Figure 7d) as in Figure 7c. The calcium release portion of the calcium signal falls off more quickly upon superposition of CF and PF stimuli than supralinear calcium release from PF stimulus alone (Figure 7e). This can be attributed to the IP3R1 bell-shaped curve response to calcium concentration [75], where the calcium concentration rises sufficiently during combine $\mathrm{Ca}^{2+}$ influx and current injection to significantly populate the inhibited state of IP3R1. Since the calcium response falls off during this sustained potion of the overall $\mathrm{Ca}^{2+}$ signal (Figure 7e), the BK conductance also falls off (Figure 7f), thus affecting the membrane potential. Figure 7 suggests that the overall contour of the calcium signal and the resulting $\mathrm{K}_{\mathrm{Ca}}$ conductances changes may underlie the effect of calcium on membrane excitability.

Taken together, these results suggest that in Purkinje neurons, coincident activation of the Purkinje spine by current injection at the soma and IP3R1-mediated calcium release with or without concurrent CF stimulus can alter membrane excitability. The results indicate that $\mathrm{K}_{\mathrm{Ca}}$ activity increased by calcium release led to earlier action potential spikes than in the absence of biochemically induced calcium transients. This is reminiscent of a study by Sausbier et al. [65] in which control mice with wild type levels of BK activation showed more spontaneous discharge relative to ataxic $\mathrm{BK}^{-/-}$mice. This is presumably due to another important result from that study: BK activity generates afterhyperpolarization of sodium (action potential) spikes. Thus, augmented BK channel activity polarizes the plasma membrane and increases excitability by more quickly resetting the sodium channels.

Computational models that combine the cell biology of calcium signaling with membrane electrophysiology involving calcium channels and calcium-activated ion channels can reveal emergent properties that are not observed in models of purely biochemical or purely electrophysiological models. The models that are presented in this work can be useful for studying these emergent properties in normal physiological Purkinje neurons, as well as in ataxia. Future studies can be developed to study spontaneous Purkinje neuron firing rate or precision of spike timing and interspike intervals in various classes of ataxias with mutations in genes that code for calcium channels [9,76-78] and potassium channels [65,79], as well as IP3R1 or related proteins (including SCA1, SCA2, SCA3, SCA15/16) [3,4,6,7,9-16].

\section{Discussion}

We determined that the Virtual Cell modeling environment [36] can be used to study cellular dynamics associated with disease states, particularly spinocerebellar ataxias with altered IP3R1 abundance or sensitivity or both. We modified a quantitative multicompartmental 
model recently developed in Virtual Cell to investigate the coupling of detailed biochemistry of IP3 and calcium signaling with membrane potential. Results show that computational modeling allows us to simulate the behavior of calcium dynamics and membrane potentials in neurons at the level of the spine and of the soma, in response to various stimuli. Our models include a myriad of ion channels and molecules important for normal Purkinje neuron function. They merge features of IP3 production, phosphoinositide and calcium signaling with conceptual representations of parallel fiber and climbing fiber stimulation, local PIP2 synthesis and sequestration, PIP2 hydrolysis, calcium influx, coincident detection at IP3R1, modulation of IP3R1 abundance and sensitivity, IP3R1-mediated supralinear calcium release, and components of membrane electrophysiology into comprehensive models that can have compartmental and spatial counterparts.

Results suggest that ICpeptides may be used to modulate calcium release in various IP3R1-associated ataxias (ataxias in which IP3R1 is less abundant, e.g., SCA15/ SCA16 as well as SCA1 and SCA3, or more sensitive than in wild type mice or unaffected individuals, e.g., SCA2 and SCA3 and likely SCA1). Simulations predict that pathophysiology associated with decreases in IP3R1 abundance can be overcome by adjusting IP3R1 sensitivity to activation by IP3, and that targeting PP1 $\alpha$ can restore normal calcium transients. Various concentrations of ICpeptide may be needed to increase IP3R1 sensitivity in SCA15/SCA16, based on the levels of reduced abundance. Similarly, various concentrations of ICpeptide could be needed to normalize IP3R1 sensitivity and consequently calcium release in SCA1, SCA2, and SCA3, based on the degrees to which IP3R1 sensitivity to IP3 is pathologically increased (dependent on the concentration of mutant protein and the relative balance with $\mathrm{PP} 1 \alpha$ ).

This project complements results by Hernjak et al. from a study on Purkinje spine calcium signaling that considered the importance of low sensitivity and high abundance of IP3R1 suggested by experiments [35]. Findings from that study suggested that increasing IP3R1 sensitivity to IP3 could not rescue calcium release in the context of low IP3. However, our results show that increased sensitivity can restore normal IP3 response if the abundance is not too low. This provides further insight into the roles that IP3R1 abundance and sensitivity play in normal cerebellar functioning and coincident detection at IP3R1. IP3R1 abundance and sensitivity can each function as the primary pathology in IP3R1-associated ataxias or can serve to partially or wholly compensate the effects of pathology. Other important conclusions from the study by Hernjak et al. were that the low IP3R1 sensitivity observed in wild type Purkinje neurons relative to other cell types may be important to restrict biochemical signals and synaptic plasticity to one spine, and that the high IP3R1 abundance in Purkinje neurons is important for ensuring generation of a robust calcium signal in an individual spine. The current study confirms that the balance of IP3R1 abundance and sensitivity is critical for obtaining robust, but not hyperactive, calcium transients. It is possible that in ataxias with reduced abundance of IP3R1, some spines will not generate robust calcium signals, while in ataxias with supersensitive IP3R1, stimulation signals may not be confined to single spines. This could affect both compartmentalization of biochemical signals, and the influence of the biochemistry on membrane electrophysiology. Similarly, therapeutically increased IP3R1 sensitivity (by application of an appropriate ICpeptide) could potentially lead to spillover of calcium into adjacent spines, if sensitivity is not finely tuned. Modeling could thus be useful for helping to determine the adequate levels of ICpeptide to be administered experimentally.

Experimental observations in SCA1 mice appear to be paradoxical [30] (Table 1). The expression of various molecules (for example, IP3R1, Homer, and SERCA) involved in glutamatergic calcium signaling and IP3R1mediated calcium release is reduced in mice and humans with SCA1 $[9,11]$. However, IP3-induced calcium release is hyperactive in SCA1 mice [8]. Insight may be gained by examining the pathology of SCA2 and SCA3. Liu et al. [10] and Chen et al. [6] found that mutant Ataxin-2 and Ataxin-3, respectively, bind the C-terminal of IP3R1 and increase the receptor's sensitivity to activation by IP3. This corresponds to hyperactive IP3-induced calcium release in both SCA2 and SCA3. Above normal numbers of CAG repeats in SCA1, SCA2, and SCA3 give each respective mutant ataxin protein a toxic gain of function that disrupts calcium homeostasis in neurons. Thus, there may be a common mechanism underlying some of the pathology of the three polyQ ataxias. This mechanism could be binding of the polyQ-expanded protein to the C-terminal of IP3R1, thereby increasing the receptor's sensitivity to IP3. This mechanism may be extendable to a large variety of polyglutamine diseases. In fact, Liu et al. reported that the mutant Ataxin-1 protein and polyQ-expanded atrophin-1, the protein mutated in Dentatorubral-pallidoluysian atrophy (DRPLA), both associate with the C-terminal of IP3R1 [10], though IP3R1 supersensitivity has not yet been assessed experimentally in either disease. Of note, a prominent feature of DRPLA is cerebellar ataxia [80-83].

The pathological gain of function of IP3R1 sensitivity that destabilizes calcium signaling [84] is also observed in a mouse model of Huntington's disease (HD), another polyQ disease [18,52,53,85-87]. Lentiviral and adenoviral infection of affected cultured medial striatal neurons or in the striatum of the ataxic HD mice themselves with the IC10 peptide lead to reduced brain atrophy and 
improved motor coordination, respectively [53]. Perhaps similar genetic targeting of Purkinje neurons in the cerebellum of existing mouse models with IC-G2736X could mitigate the pathology of SCA1, SCA2, and SCA3. This could first be tested in in situ experiments using cerebellar slices from existing mouse models, in which mutant ataxin1 binding IP3R1 directly upstream of the PP1 $\alpha$ lpha binding site, would be close enough to preclude binding of PP1 1 lpha.

Reduced gene expression of IP3R1, Homer/MyoVa, mGluR and other molecules in SCA1 may be due to direct pathology due to the effect of the mutant proteins on transcription [7,9]; it could also be due to the tight regulation of calcium homeostasis in the cerebellar Purkinje neuron [30]. Lin et al. suggest that reduced gene expression is a part of the pathology [9], but our model indicates that with exception of the calcium buffer proteins the effect on IP3R1-mediated calcium release is partially compensatory. Using the reduced fold expression observed in SCA1, normal calcium release was restored to varying degrees, depending on how much mutant Ataxin protein was placed in the model. Reduced expression of several calcium signaling genes is also observed in some Purkinje neuron subtypes in plasma membrane calcium ATP-ase (PMCA) knockout mice [20]. This could also have the effect of partial compensation in that mouse model, since PMCA contributes to expelling calcium from the Purkinje neuron cytoplasm. 'Compensatory pathology' is thus an interesting characteristic of various ataxias, and provides a level of complexity to the study of these neurological disorders. Disruption of calcium signaling and homeostasis, whether due to reduced IP3R1 levels or supersensitive IP3R1, whether in IP3R1-associated ataxias or in other ataxias, can lead to dysfunction of Purkinje cells, and impaired long-term depression and synaptic plasticity that are involved in learning and memory.

Calcium homeostasis is critical for normal function of Purkinje neurons and is thought to be tightly regulated [30]. Downregulation of parvalbumin and calbindin could therefore lead to a loss of compensation, by destabilizing supralinear calcium release and disrupting any homeostasis achieved by downregulation of other molecules. Furthermore, it has been postulated that normal or high concentration of calcium buffer proteins have a protective role in certain neurons [63]. Vig et al. suspect that the decrease in parvalbumin expression in Purkinje cells from SCA1 patients may reflect alterations in a regulatory biochemical pathway that may be important for neuronal survival [63]. Further, our model suggests that these changes (downregulation of various calcium channels and buffers) alone may not be sufficient to reproduce elevated calcium response as observed experimentally in SCA1 mice, without assuming concurrent supersensitive IP3R1.
Purkinje neurons are largely spared in SCA3 [88], while nerve cells in the pons and substantia nigra are substantially damaged. Chou et al. suggest that although prominent neuronal loss was not found in the cerebellum, the SCA3 mice displayed pronounced ataxic symptoms, suggesting that instead of neuronal demise, mutant Ataxin3 causes neuronal dysfunction and resulting ataxia [7]. While Ataxin 1 is found in the nucleus, Ataxin 2 and Ataxin3 are cytoplasmic proteins, under normal conditions. However, in brains from patients with SCA3, mutant Ataxin3 accumulates in the nucleus [28,89], as does mutant Ataxin1 [90]. Hence, results from our study of SCA1 could be extended to SCA3 modeling.

Long term feeding of SCA2 and SCA3 mice with dantrolene improved motor coordination and slowed brain atrophy $[6,10]$. Dantrolene is thought of as a 'calcium stabilizer' $[6,10,91]$ and has been shown to inhibit the ryanodine receptor (RYR), which is another calcium channel on the smooth endoplasmic reticulum [92,93]. However, all the targets of dantrolene are not known [94]. The details of the mechanism of action of dantrolene are incomplete [94], though it has been proposed as a possible therapeutic drug for SCA2 [10] and SCA3 [6]. The drug is currently approved to treat malignant hypothermia as a one-time application in response to adverse reaction due to anesthesia [94-96]. It is also used to reduce muscle spasticity in patients with neurological incidents or disorders. However, dantrolene leads to fluid buildup in the lungs, among other adverse effects [94]. Yet, considering the disturbed neuronal calcium signaling observed in polyQ ataxias, it is likely that inhibiting or downregulating either or both of the sER intracellular calcium release channels (IP3R1 and RYR) should attenuate IP3R1-mediated supralinear calcium release into the cytosol and thereby alleviate SCA2 and SCA3. This is particularly so, since both the RYR and IP3R1 channels increase in function when directly bound by cytosolic calcium. Therefore the supralinear calcium release cascade initiated by IP3 binding IP3R1 may involve cytosolic calcium also binding RYR to increase calcium release from the sER. Adding RYR to the model in the future will facilitate further study of the signaling interactions among RYR, IP3R1, other calcium channels, and the calcium-activated potassium channels. However, it should be noted that there is evidence that RYR is localized to the dendritic shaft and is excluded from spines $[97,98]$. Yet, there is also evidence that these physically separate calcium release sites functionally interact [99].

Predictions from our computer model involving SCA15/16 could be compared with novel experiments in an existing mouse model using GST-IC4 to dissociate PP1 $\alpha$ from IP3R1 in Purkinje neurons in cerebellar slices 
from ataxic mice. This could restore normal calcium and membrane potential response in ataxic mice. For such experiments, it would be useful to select a mouse model that: (i) does not completely knock out IP3R1 expression as in the IP3R $1^{-1-}$ knockout mice, but has reduced expression of IP3R1 protein, (ii) shows motor discoordination, (iii) does not possess a mutation in neuronal IP3R1 at the preferred site of PKA phosphorylation (Ser-1755) [100], and (iv) does not possess an IP3R1 mutation in the carboxyl terminal PP1 $\alpha$-binding site (2731-2749) [43]. For assessing IP3R1 haploinsufficiency in particular, as found in SCA15 and SCA16 patients $[3,12,15,16]$, it would also be useful to select a mouse model with a large heterozygous deletion mutation in IP3R1. The IP3R1 ${ }^{+/-}$mice [2,5] fulfill all of these desired features. The IP3R $1^{\text {delta18/delta18 }}$ mice have also been suggested as a mouse model for SCA15/16 [3]. The $I T P R 1^{\text {opt/opt }}$ mouse model (see Figure $1 \mathrm{~b}$ ) shows reduced IP3R1 protein levels on Western blot, but has a mutation that deletes the preferred PKA phosphorylation site (Ser-1755) $[4,100]$ in neuronal IP3R1, and therefore violates (iv). Given that a very small region of the gene is deleted and that it occurs at such an important regulation site, we suspect that IP3R1 is dysregulated in these mice. As such, we expect that these mice possess cellular pathophysiology resulting from dysregulation in addition to effects of IP3R1 insufficiency. Consequently, it is yet unclear whether results from this study will be easily extendable to calcium signaling and membrane excitability in ITPR $1^{\text {opt/opt }}$ mice.

Results from our models suggest that IP3R1-mediated calcium release can activate voltage-gated $\mathrm{K}_{\mathrm{Ca}}$ channels and thereby alter membrane excitability in the Purkinje neuron. This can have implications for ataxias that involve disruption of intracellular calcium homeostasis. It is possible that pathological alterations in calcium transients result in pathological activation of BK channels. This could lead to variations in the timing of action potentials and other electrophysiological events in the Purkinje neuron, which controls modulation of neurons downstream of the cerebellum.

\section{Conclusions}

In summary, we created a series of models incorporating biochemistry and electrophysiology that unify observations in various SCAs. These models employ several novel concepts and approaches and provide a framework for the study not only of IP3R1-associated ataxias, but of various SCAs involving mutations of other molecules in the model, such as potassium channels $[65,79,101,102]$ and calcium channels $[29,76,77,103]$.

Model results indicate that, in mouse models of various ataxias associated with activity of the calcium channel IP3R1, ICpeptides may be used to stabilize intracellular calcium concentration. Further, restoration of normal calcium release in the model does not alter fine-tuning of coincidence detection suggested by Brown et al. [42]. The hypothesis of IP3R1 supersensitivity in SCA1 is supported by simulation results. Even more, IP3R1 downregulation experimentally observed in SCA1 mice may partially compensate for the receptor's supersensitivity. Homer and MyoVa downregulation are further compensatory. However, downregulation of calcium buffer proteins accelerates pathology. The model demonstrates that IP3-mediated calcium release in the Purkinje neuron could activate voltage-gated $\mathrm{K}_{\mathrm{Ca}}$ channels, namely BK and $\mathrm{IK}$, and provides insight into the interplay between IP3R1 sensitivity and abundance in the function and dysfunction of the Purkinje cell. Results help to explain experimental findings in mice, and can be used to make predictions for further experiments, which may ultimately be translated to ataxic individuals with reduced IP3R1 protein levels or increased sensitivity. IP3R1 abundance and sensitivity are components involved in calcium signaling, but by no means the only factors involved in the signaling systems of these SCAs.

\section{Competing interests}

The authors declare that they have no competing interests.

\section{Authors' contributions}

All authors designed and implemented the models and virtual experiments. All authors wrote the manuscript. All authors read and approved the final manuscript.

\section{Acknowledgements}

We thank Dr. James Schaff, Dr. Ion Moraru, Fei Gao, and Anuradha Lakshminarayana for support in various aspects of this work. We are grateful to Dr. Arthur Hand for help with analysis of published electron microscope images, and to Dr. Louise McCullough for reading the manuscript. We are also pleased to acknowledge Dr. Ann Cowan, Dr. Raquell Holmes, and other members of the R. D. Berlin Center for Cell Analysis \& Modeling at the University of Connecticut Health Center for helpful discussions.

This project was supported by grants from the National Center for Research Resources (2P41RR013186-15) and the National Institute of General Medical Sciences (9 P41 GM103313-15) from the National Institutes of Health.

Received: 11 March 2012 Accepted: 16 May 2012

Published: 15 June 2012

\section{References}

1. Goetz CG: Textbook of Clinical Neurology. St. Louis, MO: WB Saunders; 2003.

2. Matsumoto M, Nakagawa T, Inoue T, Nagata E, Tanaka K, Takano H, Minowa O, Kuno J, Sakakibara S, Yamada M, et al: Ataxia and epileptic seizures in mice lacking type 1 inositol 1,4,5-trisphosphate receptor. Nature 1996, 379:168-171.

3. van de Leemput J, Chandran J, Knight M, Holtzclaw L, Scholz S, Cookson M, Houlden H, Gwinn-Hardy K, Fung H, Lin X, et al: Deletion at ITPR1 underlies ataxia in mice and spinocerebellar ataxia 15 in humans. PLOS Genet 2007, 3:e108.

4. Street $\mathrm{V}$, Bosma $\mathrm{M}$, Demas $\mathrm{V}$, Regan $\mathrm{M}$, Lin $\mathrm{D}$, Robinson $\mathrm{L}$, Agnew $\mathrm{W}$, Tempel B: The type 1 inositol 1,4,5-trisphosphate receptor gene is altered in the opisthotonos mouse. J Neurosci 1997, 17:635-645.

5. Ogura H, Matsumoto M, Mikoshiba K: Motor discoordination in mutant mice heterozygous for the type 1 inositol 1,4,5-trisphosphate receptor. Behav Brain Res 2001, 122:215-219. 
6. Chen X, Tang T, Tu H, Nelson O, Pook M, Hammer R, Nukina N, Bezprozvanny I: Deranged calcium signaling and neurodegeneration in spinocerebellar ataxia type 3. J Neurosci 2008, 28:12713-12724.

7. Chou A, Yeh T, Ouyang P, Chen Y, Chen S, Wang H: Polyglutamine-expanded ataxin-3 causes cerebellar dysfunction of SCA3 transgenic mice by inducing transcriptional dysregulation. Neurobiol Dis 2008, 31:89-101.

8. Inoue T, Lin X, Kohlmeier K, Orr H, Zoghbi H, Ross W: Calcium dynamics and electrophysiological properties of cerebellar Purkinje cells in SCA1 transgenic mice. J Neurophysiol 2001, 85:1750-1760.

9. Lin X, Antalffy B, Kang D, Orr H, Zoghbi H: Polyglutamine expansion down-regulates specific neuronal genes before pathologic changes in SCA1. Nat Neurosci 2000, 3:157-163.

10. Liu J, Tang T, Tu H, Nelson O, Herndon E, Huynh D, Pulst S, Bezprozvanny I: Deranged calcium signaling and neurodegeneration in spinocerebellar. ataxia type 2. J Neurosci 2009, 29:9148-9162.

11. Serra H, Byam C, Lande J, Tousey S, Zoghbi H, Orr H: Gene profiling links SCA1 pathophysiology to glutamate signaling in Purkinje cells of transgenic mice. Hum Mol Genet 2004, 13:2535-2543.

12. Iwaki A, Kawano Y, Miura S, Shibata H, Matsuse D, Li W, Furuya H, Ohyagi Y, Taniwaki T, Kira J, Fukumaki Y: Heterozygous deletion of ITPR1, but not SUMF1, in spinocerebellar ataxia type 16. J Med Genet 2008, 45:32-35.

13. Desaiah D, Vig P, Subramony S, Currier R: Inositol 1,4,5-trisphosphate receptors and protein kinase $C$ in olivopontocerebellar atrophy. Brain Res 1991, 552:36-40.

14. Zecevic N, Milosevic A, Ehrlich B: Calcium signaling molecules in human cerebellum at midgestation and in ataxia. Early Hum Dev 1999, 54:103-116.

15. Di Gregorio E, Orsi L, Godani M, Vaula G, Jensen S, Salmon E, Ferrari G, Squadrone S, Abete M, Cagnoli C, et al: Two Italian Families with ITPR1 Gene Deletion Presenting a Broader Phenotype of SCA15. Cerebellum 2010, 9:115-123.

16. Hara K, Shiga A, Nozaki H, Mitsui J, Takahashi Y, Ishiguro H, Yomono H, Kurisaki H, Goto J, Ikeuchi T, et al: Total deletion and a missense mutation of ITPR1 in Japanese SCA15 families. Neurology 2008, 71:547-551.

17. Novak M, Davis M, Li A, Goold R, Tabrizi S, Sweeney M, Houlden H, Treacy C, Giunti P: PAW32 ITPR1 gene deletion causes spinocerebellar ataxia 15/16: a genetic, clinical and radiological description of a novel kindred. J Neurol Neurosurg Psychiatry 2010, 81:e32.

18. Bezprozvanny I: Role of Inositol 1,4,5-Trishosphate Receptors in Pathogenesis of Huntington's Disease and Spinocerebellar Ataxias. Neurochem Res 2011, 36:1186-1197.

19. Schorge S, van de Leemput J, Singleton A, Houlden H, Hardy J: Human ataxias: a genetic dissection of inositol triphosphate receptor (ITPR1)-dependent signaling. Trends Neurosci 2010, 33:211-219.

20. Kurnellas M, Lee A, Li H, Deng L, Ehrlich D, Elkabes S: Molecular alterations in the cerebellum of the plasma membrane calcium ATPase 2 (PMCA2)-null mouse indicate abnormalities in Purkinje neurons. Mol Cell Neurosci 2007, 34:178-188.

21. Tu H, Tang T, Wang Z, Bezprozvanny l: Association of type 1 inositol 1,4,5-trisphosphate receptor with AKAP9 (Yotiao) and protein kinase A. J Biol Chem 2004, 279:19375-19382

22. Bezprozvanny I: The inositol 1,4,5-trisphosphate receptors. Cell Calcium 2005, 38:261-272.

23. Burright $E$, Clark $H$, Servadio A, Matilla $T$, Feddersen $R$, Yunis W, Duvick $L$, Zoghbi H, Orr H: SCA1 transgenic mice: a model for neurodegeneration caused by an expanded CAG trinucleotide repeat. Cell 1995, 82:937-948.

24. Orr H, Chung M, Banfi S, Kwiatkowski TJ, Servadio A, Beaudet A, McCall A, Duvick L, Ranum L, Zoghbi H: Expansion of an unstable trinucleotide CAG repeat in spinocerebellar ataxia type 1. Nat Genet 1993, 4:221-226.

25. Pulst S, Nechiporuk A, Nechiporuk T, Gispert S, Chen X, Lopes-Cendes I, Pearlman S, Starkman S, Orozco-Diaz G, Lunkes A, et al: Moderate expansion of a normally biallelic trinucleotide repeat in spinocerebellar ataxia type 2. Nat Genet 1996, 14:269-276.

26. Boy J, Schmidt T, Schumann U, Grasshoff U, Unser S, Holzmann C, Schmitt I, Karl T, Laccone F, Wolburg H, et al: A transgenic mouse model of spinocerebellar ataxia type 3 resembling late disease onset and gender-specific instability of CAG repeats. Neurobiol Dis 2010, 37:284-293.

27. Dürr A, Stevanin G, Cancel G, Duyckaerts C, Abbas N, Didierjean O, Chneiweiss H, Benomar A, Lyon-Caen O, Julien J, et al: Spinocerebellar ataxia 3 and Machado-Joseph disease: clinical, molecular, and neuropathological features. Ann Neurol 1996, 39:490-499.
28. Paulson H, Perez M, Trottier Y, Trojanowski J, Subramony S, Das S, Vig P, Mandel J, Fischbeck K, Pittman R: Intranuclear inclusions of expanded polyglutamine protein in spinocerebellar ataxia type 3. Neuron 1997, 19:333-344.

29. Mantuano E, Veneziano L, Spadaro M, Giunti P, Guida S, Leggio M, Verriello L, Wood N, Jodice C, Frontali M: Clusters of non-truncating mutations of P/Q type Ca2+ channel subunit Ca(v)2.1 causing episodic ataxia 2. $J$ Med Genet 2004, 41:e82.

30. Inoue T: Dynamics of calcium and its roles in the dendrite of the cerebellar Purkinje cell. Keio J Med 2003, 52:244-249.

31. Gardner R: "SCA16" is really SCA15. J Med Genet 2008, 45:192.

32. Brown S-A, Loew LM: Toward A Computational Model Of IP3R1associated Ataxia. Biophysical Journal 2009, 96(3:S1):96a.

33. Khodakhah K, Ogden D: Functional heterogeneity of calcium release by inositol trisphosphate in single Purkinje neurones, cultured cerebellar astrocytes, and peripheral tissues. Proc Natl Acad Sci U S A 1993, 90:4976-4980

34. Fujiwara A, Hirose K, Yamazawa T, lino M: Reduced IP3 sensitivity of IP3 receptor in Purkinje neurons. Neuroreport 2001, 12:2647-2651.

35. Hernjak N, Slepchenko B, Fernald K, Fink C, Fortin D, Moraru I, Watras J, Loew L: Modeling and analysis of calcium signaling events leading to long-term depression in cerebellar Purkinje cells. Biophys J 2005, 89:3790-3806

36. Brown SA, Moraru II, Schaff JC, Loew LM: Virtual NEURON: a strategy for merged biochemical and electrophysiological modeling. J Comput Neurosci 2011, 31:385-400.

37. Loew L, Schaff J: The virtual cell: a software environment for computational cell biology. Trends Biotechnol 2001, 19:401-406.

38. Schaff J, Fink C, Slepchenko B, Carson J, Loew L: A general computational framework for modeling cellular structure and function. Biophys J 1997, 73:1135-1146.

39. Schaff J, Slepchenko B, Loew L: Physiological modeling with virtual cell framework. Methods Enzymol 2000, 321:1-23.

40. Cowan AE, Moraru II, Schaff JC, Slepchenko BM, Loew LM: Spatial modeling of cell signaling networks. Methods Cell Biol 2012, 110:195-221.

41. Moraru I, Schaff J, Slepchenko B, Loew L: The virtual cell: an integrated modeling environment for experimental and computational cell biology. Ann N Y Acad Sci 2002, 971:595-596.

42. Brown S, Morgan F, Watras J, Loew L: Analysis of phosphatidylinositol-4,5bisphosphate signaling in cerebellar Purkinje spines. Biophys J 2008, 95:1795-1812

43. Tang T, Tu H, Wang Z, Bezprozvanny I: Modulation of type 1 inositol $(1,4,5)$-trisphosphate receptor function by protein kinase a and protein phosphatase 1alpha. J Neurosci 2003, 23:403-415.

44. Wang S, Denk W, Häusser M: Coincidence detection in single dendritic spines mediated by calcium release. Nat Neurosci 2000, 3:1266-1273.

45. Finch $E$, Augustine G: Local calcium signalling by inositol-1,4,5trisphosphate in Purkinje cell dendrites. Nature 1998, 396:753-756.

46. Takechi H, Eilers J, Konnerth A: A new class of synaptic response involving calcium release in dendritic spines. Nature 1998, 396:757-760.

47. Destexhe A, Babloyantz A, Sejnowski T: Ionic mechanisms for intrinsic slow oscillations in thalamic relay neurons. Biophys J 1993, 65:1538-1552.

48. De Schutter $\mathrm{E}$, Bower J: An active membrane model of the cerebellar Purkinje cell. I. Simulation of current clamps in slice. J Neurophysiol 1994, 71:375-400.

49. Miyasho T, Takagi H, Suzuki H, Watanabe S, Inoue M, Kudo Y, Miyakawa H: Low-threshold potassium channels and a low-threshold calcium channel regulate $\mathrm{Ca} 2+$ spike firing in the dendrites of cerebellar Purkinje neurons: a modeling study. Brain Res 2001, 891:106-115.

50. Gabbiani F, Midtgaard J, Knöpfel T: Synaptic integration in a model of cerebellar granule cells. J Neurophysiol 1994, 72:999-1009.

51. Cui J, Yang H, Lee U: Molecular mechanisms of BK channel activation. Cell Mol Life Sci 2009, 66:852-875.

52. Tang $T$, Tu H, Chan E, Maximov A, Wang Z, Wellington C, Hayden M Bezprozvanny I: Huntingtin and huntingtin-associated protein 1 influence neuronal calcium signaling mediated by inositol- $(1,4,5)$ triphosphate receptor type 1. Neuron 2003, 39:227-239.

53. Tang T, Guo C, Wang H, Chen X, Bezprozvanny I: Neuroprotective effects of inositol 1,4,5-trisphosphate receptor C-terminal fragment in a Huntington's disease mouse model. J Neurosci 2009, 29:1257-1266 
54. Brown SA, Holmes RM, Loew LM: Spatial Organization and Diffusion in Neuronal Signaling. In Computational Systems Neurobiology. Edited by Le Novère N. Dordrecht: Springer; 2012. Chapter 5, in press.

55. Tu H, Wang Z, Bezprozvanny I: Modulation of mammalian inositol 1,4,5-trisphosphate receptor isoforms by calcium: a role of calcium sensor region. Biophys J 2005, 88:1056-1069.

56. Sarkisov D, Wang S: Order-dependent coincidence detection in cerebellar Purkinje neurons at the inositol trisphosphate receptor. J Neurosci 2008, 28:133-142.

57. Kasumu A, Bezprozvanny I: Deranged Calcium Signaling in Purkinje Cells and Pathogenesis in Spinocerebellar Ataxia 2 (SCA2) and Other Ataxias. Cerebellum 2010, doi:10.1007/s12311-010-0182-9.

58. Miyata M, Finch E, Khiroug L, Hashimoto K, Hayasaka S, Oda S, Inouye M, Takagishi Y, Augustine G, Kano M: Local calcium release in dendritic spines required for long-term synaptic depression. Neuron 2000, 28:233-244.

59. Wagner W, Hammer Jr: Myosin V and the endoplasmic reticulum: the connection grows. J Cell Biol 2003, 163:1193-1196.

60. Vecellio M, Schwaller B, Meyer M, Hunziker W, Celio M: Alterations in Purkinje cell spines of calbindin D-28 $\mathrm{k}$ and parvalbumin knock-out mice. Eur J Neurosci 2000, 12:945-954.

61. Vig PJ, Wei J, Shao Q, Lopez ME, Halperin R, Gerber J: Suppression of Calbindin-D28k Expression Exacerbates SCA1 Phenotype in a Disease Mouse Model. Cerebellum 2011, doi:10.1007/s12311-011-0323-9.

62. Schmidt H, Stiefel K, Racay P, Schwaller B, Eilers J: Mutational analysis of dendritic Ca2+ kinetics in rodent Purkinje cells: role of parvalbumin and calbindin D28k. J Physiol 2003, 551:13-32.

63. Vig P, Subramony S, Burright E, Fratkin J, McDaniel D, Desaiah D, Qin Z: Reduced immunoreactivity to calcium-binding proteins in Purkinje cells precedes onset of ataxia in spinocerebellar ataxia-1 transgenic mice. Neurology 1998, 50:106-113.

64. Vig P, Subramony S, McDaniel D: Calcium homeostasis and spinocerebellar ataxia-1 (SCA-1). Brain Res Bull 2001, 56:221-225.

65. Sausbier M, Hu H, Arntz C, Feil S, Kamm S, Adelsberger H, Sausbier U, Sailer C, Feil R, Hofmann F, et al: Cerebellar ataxia and Purkinje cell dysfunction caused by $\mathrm{Ca} 2+-$ activated $\mathrm{K}+$ channel deficiency. Proc Natl Acad Sci US A 2004, 101:9474-9478.

66. Cheron G, Sausbier M, Sausbier U, Neuhuber W, Ruth P, Dan B, Servais L: BK channels control cerebellar Purkinje and Golgi cell rhythmicity in vivo. PLoS One 2009, 4:e7991.

67. Kent J, Meredith A: BK channels regulate spontaneous action potential rhythmicity in the suprachiasmatic nucleus. PLoS One 2008, 3:e3884.

68. Womack M, Khodakhah K: Dendritic control of spontaneous bursting in cerebellar Purkinje cells. J Neurosci 2004, 24:3511-3521.

69. Walter J, Alviña K, Womack M, Chevez C, Khodakhah K: Decreases in the precision of Purkinje cell pacemaking cause cerebellar dysfunction and ataxia. Nat Neurosci 2006, 9:389-397.

70. Oliver D, Taberner A, Thurm H, Sausbier M, Arntz C, Ruth P, Fakler B, Liberman M: The role of BKCa channels in electrical signal encoding in the mammalian auditory periphery. J Neurosci 2006, 26:6181-6189.

71. Weaver A, Olsen M, McFerrin M, Sontheimer $H$ : BK channels are linked to inositol 1,4,5-triphosphate receptors via lipid rafts: a novel mechanism for coupling $[\mathrm{Ca}(2+)](\mathrm{i})$ to ion channel activation. J Biol Chem 2007, 282:31558-31568.

72. Dai S, Hall D, Hell J: Supramolecular assemblies and localized regulation of voltage-gated ion channels. Physiol Rev 2009, 89:411-452.

73. Sakagami Y, Yamamoto K, Sugiura S, Inokuchi K, Hayashi T, Kato N: Essential roles of Homer-1a in homeostatic regulation of pyramidal cell excitability: a possible link to clinical benefits of electroconvulsive shock. Eur J Neurosci 2005, 21:3229-3239.

74. Vaithianathan T, Bukiya A, Liu J, Liu P, Asuncion-Chin M, Fan Z, Dopico A: Direct regulation of BK channels by phosphatidylinositol 4,5bisphosphate as a novel signaling pathway. J Gen Physiol 2008, 132:13-28.

75. Bezprozvanny I, Watras J, Ehrlich B: Bell-shaped calcium-response curves of Ins(1,4,5)P3- and calcium-gated channels from endoplasmic reticulum of cerebellum. Nature 1991, 351:751-754.

76. Guida S, Trettel F, Pagnutti S, Mantuano E, Tottene A, Veneziano L, Fellin T, Spadaro M, Stauderman K, Williams M, et al: Complete loss of P/Q calcium channel activity caused by a CACNA1A missense mutation carried by patients with episodic ataxia type 2. Am J Hum Genet 2001, 68:759-764.
77. Zhuchenko O, Bailey J, Bonnen P, Ashizawa T, Stockton D, Amos C, Dobyns W, Subramony S, Zoghbi H, Lee C: Autosomal dominant cerebellar ataxia (SCA6) associated with small polyglutamine expansions in the alpha 1A-voltage-dependent calcium channel. Nat Genet 1997, 15:62-69.

78. Yue Q, Jen J, Nelson S, Baloh R: Progressive ataxia due to a missense mutation in a calcium-channel gene. Am J Hum Genet 1997, 61:1078-1087.

79. Imbrici P, Cusimano A, D'Adamo M, De Curtis A, Pessia M: Functional characterization of an episodic ataxia type-1 mutation occurring in the S1 segment of hKv1.1 channels. Pflugers Arch 2003, 446:373-379.

80. Adachi N, Arima K, Asada T, Kato M, Minami N, Goto Yi, Onuma T, Ikeuchi T, Tsuji S, Hayashi M, Fukutani Y: Dentatorubral-pallidoluysian atrophy (DRPLA) presenting with psychosis. J Neuropsychiatry Clin Neurosci 2001, 13:258-260

81. Martins S, Matamá T, Guimarães L, Vale J, Guimarães J, Ramos L, Coutinho P, Sequeiros J, Silveira I: Portuguese families with dentatorubropallidoluysian atrophy (DRPLA) share a common haplotype of Asian origin. Eur J Hum Genet 2003, 11:808-811.

82. Yabe I, Sasaki H, Kikuchi S, Nonaka M, Moriwaka F, Tashiro K: Late onset ataxia phenotype in dentatorubro-pallidoluysian atrophy (DRPLA). J Neurol 2002, 249:432-436.

83. Naito H, Oyanagi S: Familial myoclonus epilepsy and choreoathetosis: hereditary dentatorubral-pallidoluysian atrophy. Neurology 1982 32:798-807.

84. Bezprozvanny I: Calcium signaling and neurodegenerative diseases. Trends Mol Med 2009, 15:89-100.

85. Bezprozvanny I, Hayden M: Deranged neuronal calcium signaling and Huntington disease. Biochem Biophys Res Commun 2004, 322:1310-1317.

86. Zhang H, Li Q, Graham R, Slow E, Hayden M, Bezprozvanny I: Full length mutant huntingtin is required for altered $\mathrm{Ca} 2+$ signaling and apoptosis of striatal neurons in the YAC mouse model of Huntington's disease. Neurobiol Dis 2008, 31:80-88.

87. Bezprozvanny I: Inositol 1,4,5-tripshosphate receptor, calcium signalling and Huntington's disease. Subcell Biochem 2007, 45:323-335.

88. Stevanin G, Dürr A, Brice A: Clinical and molecular advances in autosomal dominant cerebellar ataxias: from genotype to phenotype and physiopathology. Eur J Hum Genet 2000, 8:4-18.

89. Schmidt T, Lindenberg K, Krebs A, Schöls L, Laccone F, Herms J, Rechsteiner M, Riess $\mathrm{O}$, Landwehrmeyer $\mathrm{G}$ : Protein surveillance machinery in brains with spinocerebellar ataxia type 3: redistribution and differential recruitment of $26 \mathrm{~S}$ proteasome subunits and chaperones to neuronal intranuclear inclusions. Ann Neurol 2002, 51:302-310.

90. Klement I, Skinner P, Kaytor M, Yi H, Hersch S, Clark H, Zoghbi H, Orr H: Ataxin-1 nuclear localization and aggregation: role in polyglutamineinduced disease in SCA1 transgenic mice. Cell 1998, 95:41-53.

91. Makarewicz D, Ziemińska E, Łazarewicz J: Dantrolene inhibits NMDAinduced $45 \mathrm{Ca}$ uptake in cultured cerebellar granule neurons. Neurochem Int 2003, 43:273-278.

92. Zhao F, Li P, Chen S, Louis C, Fruen B: Dantrolene inhibition of ryanodine receptor $\mathrm{Ca} 2+$ release channels. Molecular mechanism and isoform selectivity. J Biol Chem 2001, 276:13810-13816.

93. Fruen B, Mickelson J, Louis C: Dantrolene inhibition of sarcoplasmic reticulum $\mathrm{Ca} 2+$ release by direct and specific action at skeletal muscle ryanodine receptors. J Biol Chem 1997, 272:26965-26971.

94. Krause T, Gerbershagen M, Fiege M, Weisshorn R, Wappler F: Dantrolene-a review of its pharmacology, therapeutic use and new developments. Anaesthesia 2004, 59:364-373.

95. Gerbershagen M, Fiege M, Krause T, Agarwal K, Wappler F: Dantrolene. Pharmacological and therapeutic aspects. Anaesthesist 2003, 52:238-245.

96. Kobayashi S, Yano M, Suetomi T, Ono M, Tateishi H, Mochizuki M, Xu X, Uchinoumi H, Okuda S, Yamamoto T, et al: Dantrolene, a therapeutic agent for malignant hyperthermia, markedly improves the function of failing cardiomyocytes by stabilizing interdomain interactions within the ryanodine receptor. J Am Coll Cardiol 2009, 53:1993-2005.

97. Sharp AH, MCPherson PS, Dawson TM, Aoki C, Campbell KP, Snyder SH: Differential immunohistochemical localization of inositol 1,4,5trisphosphate- and ryanodine-sensitive $\mathrm{Ca} 2+$ release channels in rat brain. J Neurosci 1993, 13:3051-3063.

98. Walton PD, Airey JA, Sutko JL, Beck CF, Mignery GA, Südhof TC, Deerinck TJ, Ellisman $\mathrm{MH}$ : Ryanodine and inositol trisphosphate receptors coexist in avian cerebellar Purkinje neurons. J Cell Biol 1991, 113:1145-1157. 
99. Khodakhah K, Armstrong CM: Inositol trisphosphate and ryanodine receptors share a common functional Ca2+ pool in cerebellar Purkinje neurons. Biophys J 1997, 73:3349-3357.

100. Ln W, Li W, Yule D: Phosphorylation of type-1 inositol 1,4,5-trisphosphate receptors by cyclic nucleotide-dependent protein kinases: a mutational analysis of the functionally important sites in the S2+ and S2- splice variants. J Biol Chem 2003, 278:45811-45817.

101. Imbrici P, D'Adamo M, Cusimano A, Pessia M: Episodic ataxia type 1 mutation F184C alters Zn2 +-induced modulation of the human K+ channel Kv1.4-Kv1.1/Kvbeta1.1. Am J Physiol Cell Physiol 2007, 292:C778-787.

102. Imbrici P, D'Adamo M, Kullmann D, Pessia M: Episodic ataxia type 1 mutations in the KCNA1 gene impair the fast inactivation properties of the human potassium channels Kv1.4-1.1/Kvbeta1.1 and Kv1.4-1.1/Kvbeta1.2. Eur J Neurosci 2006, 24:3073-3083.

103. Tonelli A, D'Angelo M, Salati R, Villa L, Germinasi C, Frattini T, Meola G, Turconi A, Bresolin N, Bassi M: Early onset, non fluctuating spinocerebellar ataxia and a novel missense mutation in CACNA1A gene.

J Neurol Sci 2006, 241:13-17.

doi:10.1186/1752-0509-6-70

Cite this article as: Brown and Loew: Computational analysis of calcium signaling and membrane electrophysiology in cerebellar Purkinje neurons associated with ataxia. BMC Systems Biology 2012 6:70.

\section{Submit your next manuscript to BioMed Central and take full advantage of:}

- Convenient online submission

- Thorough peer review

- No space constraints or color figure charges

- Immediate publication on acceptance

- Inclusion in PubMed, CAS, Scopus and Google Scholar

- Research which is freely available for redistribution 